\title{
HOMOGENIZATION OF STOCHASTIC SEMILINEAR PARABOLIC EQUATIONS WITH NON-LIPSCHITZ FORCINGS IN DOMAINS WITH FINE GRAINED BOUNDARIES*
}

\author{
MAMADOU SANGO ${ }^{\dagger}$
}

\begin{abstract}
The present work deals with the homogenization and in-depth asymptotic analysis of a nonlinear stochastic evolution equation with non-Lipschitz nonlinearities in a domain with fine grained boundaries in which the obstacles have a non-periodic distribution. Under appropriate conditions on the data it is proved that a solution of the initial problem converges in suitable topologies to a solution of a limit problem which contains an additional term of capacity type. The notion of solution is that of weak probabilistic which is a system consisting of a probability space, Wiener process, and a solution in the distribution sense of the problem.
\end{abstract}

Key words. Stochastic partial differential equation, homogenization, perforated domains.

Mathematics Subject Classification. 60H15, 35B27.

\section{Introduction}

Let $\Omega$ be a bounded domain in the Euclidean space $\mathbf{R}^{n}(n \geq 3)$ with boundary $\partial \Omega$. For $0<T<\infty$, we denote by $Q_{T}$ the cylinder $(0, T) \times \Omega$. We assume that for each natural number $s$ there is defined a finite number of disjoint closed sets $F_{i}^{(s)}(i=$ $1,2, \ldots, I(s), I(s)$ is a natural number) lying inside $\Omega$. Let $\Omega^{(s)}$ be the sequence of perforated domains obtained by removing the set $F^{(s)}=\cup_{i=1}^{I(s)} F_{i}^{(s)}$ from $\Omega$, i.e., $\Omega^{(s)}=$ $\Omega \backslash F^{(s)}$; the boundary $\partial \Omega^{(s)}$ of $\Omega^{(s)}$ is assumed to be sufficiently smooth (e.g., of class $C^{2}$ ). In the sequel we shall formulate some conditions on $F_{i}^{(s)}$ from which it follows in particular that as $s \rightarrow \infty$ the diameters of $F_{i}^{(s)}$ tend to zero. We consider the sequence of cylindrical domains $Q_{T}^{(s)}=(0, T) \times \Omega^{(s)}$. In $Q_{T}^{(s)}$, we consider the initial boundary value problem for the stochastic partial differential equation

$$
\begin{gathered}
d u^{(s)}=\left[\Delta u^{(s)}+f\left(t, u^{(s)}\right)\right] d t+\sum_{k=1}^{d} g_{k}\left(t, u^{(s)}\right) d w_{t}^{(s) k}, \text { in } Q_{T}^{(s)} \\
u^{(s)}(t, x)=0, \text { on } \partial Q_{T}^{(s)} \\
u^{(s)}(0, x)=u_{0}(x), \text { in } \Omega^{(s)}
\end{gathered}
$$

where $\partial Q_{T}^{(s)}=(0, T) \times \partial \Omega^{(s)}, f$ and $g_{k}$ are real valued functions taking values in some Hilbert spaces, $u_{0}(x)$ is a deterministic real valued function defined on $\Omega^{(s)}, \Delta$ is the Laplace operator, i.e., $\Delta=\sum_{i=1}^{n} \partial^{2} / \partial x_{i}^{2}$, and $W^{(s)}(t)=\left(w_{t}^{(s) 1}, \ldots, w_{t}^{(s) d}\right)$ is a $d-$ dimensional Wiener process.

The case of linear noises which corresponds to $f$ and $g^{k}$ independent of $u^{(s)}$ was considered in [50]. The present paper considers the more difficult question of nonlinear noise which in addition does not satisfy the Lipschitz condition. In this regard the

${ }^{*}$ Received: December 30, 2011; accepted (in revised form): February 5, 2013. Communicated by Weinan E.

${ }^{\dagger}$ Department of Mathematics and Applied Mathematics University of Pretoria, Private bag X20 Hatfield 0028 PRETORIA, South Africa (sango1767@gmail.com; mamadou.sango@up.ac.za). 
notion of solution for the problem (1.1)-(1.3) is already different from the one in [50]. As is known the adequate notion of solution here is that of probabilistic weak solution

$$
\left(\mathcal{S}^{(s)}, \mathcal{F}^{(s)},\left\{\mathcal{F}_{t}^{(s)}\right\}_{0 \leq t \leq T}, \mathcal{P}^{(s)}, W^{(s)}, u^{(s)}\right),
$$

which will be defined in the next section.

The aim of the present work is to investigate the behavior of the sequence

$$
\left(\mathcal{S}^{(s)}, \mathcal{F}^{(s)},\left\{\mathcal{F}_{t}^{(s)}\right\}_{0 \leq t \leq T}, \mathcal{P}^{(s)}, W^{(s)}, u^{(s)}\right), s=1,2, \ldots
$$

of solutions of problem (1.1)-(1.3) under some appropriate conditions on the data and on the perforated domain $\Omega^{(s)}$ that we make precise later on. We shall prove that under those conditions the sequence of solutions converge in suitable topologies to a probabilistic system $\left(\Xi, G,\left\{G_{t}\right\}_{0 \leq t \leq T}, P, W, u\right)$ which is a weak solution of a homogenized stochastic evolution problem in the cylinder $Q_{T}$. Namely

$$
\begin{gathered}
d u=[\Delta u-c(x) u+f(t, u)] d t+\sum_{k=1}^{d} g_{k}(t, u) d w_{t}^{k} \text { in } Q_{T}, \\
u(t, x)=0 \text { on } \partial Q_{T}, \\
u(0, x)=u_{0}(x) \text { in } \Omega .
\end{gathered}
$$

The function $c(x)$ is defined in terms of the geometry of the sets $F_{i}^{(s)}$. The investigation will be based on a corrector formula with remainder term involving $u^{(s)}$ and its limits and the solutions of some auxiliary elliptic boundary value problems that we introduce in neighborhoods of the sets $F_{i}^{(s)}$, the analog of cell problems in the periodic homogenization setting. The accuracy of the corrector formula is given by proving that the remainder term converges to zero in suitable topologies. This important and crucial rigorous asymptotic study provides an in-depth analysis of the problem. A central idea in the work which is absent in the deterministic case lies in seeking the remainder term in the corrector formula of $u^{(s)}$ as a sum of stochastic integrals of some functions with respect to the Wiener processes $w_{t}^{(s) k}(k=1, \ldots, d)$. This representation is of paramount importance in achieving our goals. In view of the different notion of solution and the kind of nonlinearity considered the present work differs from [50] in many regards. The lack of appropriate regularity of the solution, such as $L_{\infty}$-boundedness, requires new ideas at various stages to deal with the arising difficulties.

Since the pioneering work of Bensoussan and Temam [4, 5] in the earlier 1970's, stochastic partial differential equations (SPDE's) have grown into one of the most active areas of research in the frontiers of mathematics today. The incorporation of randomness in partial differential equations (PDE's) governing a wide range of models arising in applied sciences make them more realistic in view of the overwhelming prevalence of stochastic factors in nature. This thought pattern based on both empirical and experimental data led to the emergence of SPDE's. A great wealth of results have been obtained in several directions of research and collected in numerous monographs. An area which has remained relatively dormant is that of perturbed SPDE's, and notably homogenization of SPDE's. We note that the interaction between probability 
theory and homogenization has been mainly in the use of the probabilistic methods in order to investigate deterministic perturbed PDE's and in the study of PDE's with rapidly oscillating random coefficients or in perforated random domains. A wealth of interesting results using various methods is now available and the field is expanding. We refer to $[7,9,11,19,24,29,31,35,36,37,43,44,45,46,48,54,55,60,65]$ for classical and recent results. It is worth noting the new direction of numerical homogenization which has also recorded in recent years some success in the treatment of random parabolic equations; see $[1,20,21]$. Further references can be found in these papers.

As far as the homogenization of stochastic evolution problems driven by random noises is concerned, very little is known so far. In view of the huge interest presented by SPDE's in applied sciences, there is a need to develop frameworks that will enable the study of the corresponding stochastic homogenization problems. The work of Bensoussan [6] which deals with the homogenization of a sequence of stochastic PDE's in a fixed domain was pioneering in this direction. The work [50] was the first dealing with the homogenization of stochastic evolution PDE's in perforated domains; furthermore no conditions of periodic distribution of the holes were assumed. These works were followed by $[27,28,62]$ and $[63]$ in the periodic setting. The two last papers treat the case of perforated domains with periodically distributed perforations. In particular they extend Tartar's energy method and Nguetseng-Allaire's two-scale convergence $([3,41])$ to the stochastic setting; a nice introduction to these notions can be found in the monograph [14]. It should be noted that the limit problem in these cases does not differ in form from the original one as in the corresponding deterministic case.

In the present work we limit ourselves to a simpler model of SPDE's which, as witnessed in the subsequent pages, encapsulates most of the complexities arising in SPDE's in domains with fine grained boundaries. We provide a rigorous investigation of the problem and lay down a framework which can be used to study more general problems. We note that the stochastic limit problem obtained in the homogenization process has an additional term of capacity type depending on the geometry of the sets $F_{i}^{(s)}$. This phenomenon arises also in deterministic problems and was originally discovered in homogenization of elliptic boundary value problems in the celebrated work of Khruslov and Marchenko [38] (see also the long awaited English version [39]); the nonlinear version of their theory was developed by Skrypnik [58]. More information on results obtained in this kind of deterministic problems can be found in, for example, [44] (heat equations in perforated domains by probabilistic means) and $[2,16,13,18,51]$. Deterministic evolution problems in non-periodically perforated domains have been less studied; we refer to $[12,15,59,52]$ for some results in this direction.

It should be noted that even under the stronger conditions of periodic distribution of holes our main result has an additional novelty compared to the previous work done in $[6,27,28,62]$ and [63] in the periodic framework, in the sense that there the authors deal only with noises that satisfy the Lipschitz condition, and we also obtain an optimal corrector result (Theorem 3.2) which was not considered in these papers. The framework developed in the paper is quite general and enables the study of wider classes of stochastic evolution problems in varying domains with nonperiodic geometry. An analog of the homogenization theory of Cioranescu and Murat elaborated in [16] can be developed in the framework of SPDE's. In view of the appearance of measures in the limit problem in that framework, a new class of SPDE's will emerge, notably SPDE's with measure-valued coefficients. However there is no 
indication that sharp corrector results as in the present work could be obtained. Still, in this direction an important issue is the derivation of Brinkman's law for turbulent flows of fluids governed by the Stochastic-Navier Stokes equations. The deterministic stationary case was solved in the work of Allaire [2]. Since homogenization of SPDE's is in its infancy, numerous problems are waiting to be solved.

It is by now well known that problems such as (1.1)-(1.3) describe processes (heat conduction, diffusion and reaction-diffusion processes) taking place in highly heterogeneous media under the influence of random factors. We can also view the problem as a simplified stochastic model of fluid dynamics in a turbulent regime filling a cylinder with tiny cylindrical obstacles.

The plan of the paper is as follows. In Section 2, we state some preliminary results on the existence and uniqueness of a solution of problem (1.1)-(1.3) following [8] and [40]. Next we introduce some auxiliary elliptic boundary value problems in the neighborhood of the sets $F_{i}^{(s)}$ whose solutions and their a priori pointwise estimates play a central role in our investigation, and we state the conditions on the geometry of the perforated set $\Omega^{(s)}$ and formulate our main result. In Section 3 , we construct a corrector formula with remainder term for $u^{(s)}$ and prove that the remainder term converges to zero in suitable topologies. In Section 4, we derive the stochastic evolution problem satisfied by the limit of $u^{(s)}$ in a sense that we make precise in Section 2.

\section{Preliminary results}

We shall use the following well-known Lebesgue and Sobolev spaces $L_{p}(\cdot), W_{p}^{1}(\cdot)$, $\stackrel{o}{W_{p}^{1}}(\cdot)(p \geq 1)$ (we denote $H^{1}(\Omega)=: W_{2}^{1}(\cdot), H_{0}^{1}(\cdot)=\stackrel{o}{W_{2}^{1}}(\cdot)$ ) as defined, for example in [22]; $H^{-1}(\cdot)$ stands for the dual of $H_{0}^{1}(\cdot),(.,$.$) denotes the scalar product in L_{2}(\cdot)$, where throughout . stands for either $\Omega$ or $\Omega^{(s)}$. $C_{o}^{\infty}(\cdot)$ is the space of infinitely differentiable functions with compact support in $\cdot$

Let $X$ be any Banach space of functions defined on $\Omega$ or $\Omega^{(s)}$ and let $(\mathcal{S}, \mathcal{F}, \mathcal{P})$ be any probability space over which the expectation is denoted by $E$. By the symbol $L_{p}\left(\mathcal{S}, L_{q}(0, T, X)\right)$ we denote the space of functions $u=u(\omega, t, x)$ defined on $\mathcal{S} \times[0, T]$ with values in $X$, and such that

a. $u(\omega, t, x)$ is measurable with respect to $(\omega, t)$ and for each $t$ is $\mathcal{F}_{t}$-measurable in $\omega$.

b. $u(\omega, t, x) \in X$ for almost all $(\omega, t)$, and

$$
\|u\|_{L_{p}\left(\mathcal{S}, L_{q}(0, T, X)\right)}=\left(E \int_{0}^{T}\left(\|u(t)\|_{X}^{q} d t\right)^{p / q}\right)^{1 / p}<\infty ;
$$

$E$ denotes the mathematical expectation. If $q=\infty$, we write

$$
\|u\|_{L_{p}\left(\mathcal{S}, L_{\infty}(0, T, X)\right)}=\left(\text { Eess } \sup _{0 \leq t \leq T}\|u(t)\|_{X}^{p}\right)^{1 / p}<\infty
$$

Let $Z$ be any Banach space of functions defined on the cylinder $Q_{T}$. We denote by $L_{p}(\mathcal{S}, Z)$ the space of random variables $u$ defined on $S$ with values in $Z$ and such that

$$
\|u\|_{L_{p}(\mathcal{S}, Z)}=\left(E\|u\|_{Z}^{p}\right)^{1 / p}<\infty .
$$

The following density result, similar in proof to the deterministic case (see e.g., [33, Chap.1, § 1.1], [64, §25]), holds. 
Lemma 2.1. Let $\Omega$ be a sufficiently smooth domain, $p, q \geq 1$, and let

$$
u \in L_{q}\left(\mathcal{S}, L_{\infty}\left(0, T, L_{p}(\Omega)\right)\right) \cap L_{q}\left(\mathcal{S}, L_{p}\left(0, T, \stackrel{o}{W_{p}^{1}}(\Omega)\right)\right) .
$$

Then there exists a sequence of functions

$$
\left(u_{m}\right)_{m=1,2, \ldots} \in L_{q}\left(\mathcal{S}, C_{0}^{\infty}\left(Q_{T}\right)\right)
$$

such that

$$
\begin{aligned}
\lim _{m \rightarrow \infty}\left\|u_{m}-u\right\|_{L_{q}\left(\mathcal{S}, L_{\infty}\left(0, T, L_{p}(\Omega)\right)\right)} & =0, \\
\lim _{m \rightarrow \infty}\left\|\frac{\partial\left(u_{m}-u\right)}{\partial x}\right\| \|_{L_{q}\left(\mathcal{S}, L_{p}\left(0, T, L_{p}(\Omega)\right)\right)} & =0 .
\end{aligned}
$$

We also have the following result, which is [34, Chap. 1, Lemma 1.3].

Lemma 2.2. Let $\left(g_{\kappa}\right)_{\kappa=1,2 \ldots}$ and $g$ be some functions in $L_{q}\left(0, T, L_{q}(\Omega)\right)$ with $q \in$ $(1, \infty)$ such that

$$
\left\|g_{\kappa}\right\|_{L_{q}\left(0, T, L_{q}(\Omega)\right)} \leq C, \forall \kappa,
$$

and as $\kappa \rightarrow \infty$

$$
g_{\kappa} \rightarrow g \text { for almost all }(t, x) \in Q_{T} .
$$

Then $g_{\kappa}$ weakly converges to $g$ in $L_{q}\left(0, T, L_{q}(\Omega)\right)$.

REMARK 2.1. The results of the lemma hold for the space $L_{q}\left(\Xi, L_{q}\left(0, T, L_{q}(\Omega)\right)\right)$ in $\boldsymbol{\Xi} \times Q_{T}$.

The next result is from [56, Section 8 , Theorem 5]. It is a sharper version of a theorem due to Aubin (cf. [34, Chap. 1, Par. 5]).

Lemma 2.3. Let $X, B$, and $Y$ be some Banach spaces such that $X$ is compactly embedded into $B$ and let $B$ be a subset of $Y$. For any $1 \leq p, q \leq \infty$, let $V$ be a set bounded in $L_{q}(0, T, X)$ such that

$$
\lim _{\theta \rightarrow 0} \int_{0}^{T-\theta}\|v(t+\theta)-v(t)\|_{Y}^{p} d t=0, \text { uniformly for all } v \in V .
$$

Then $V$ is relatively compact in $L_{p}(0, T, B)$.

The following Poincaré inequalities in an annulus as proved in [58, Lemma 1.4, Chap. 8]play a crucial role for obtaining sharp estimates.

Lemma 2.4. Let $K\left(\rho_{1}, \rho_{2}\right)$ be the ring $\left\{x: 0<\rho_{1}<|x|<\rho_{2}<r\right\}$ and $f(x) \in$ $H^{1}(B(0, r))$. Then

$$
\int_{K\left(\rho_{1}, \rho_{2}\right)}|f(x)|^{2} d x \leq C\left[\rho_{2}^{2}-\rho_{1}^{2}\right] \int_{K\left(\rho_{1}, r\right)}|\nabla f|^{2} d x+C \frac{\rho_{2}^{n}-\rho_{1}^{n}}{r^{n}} \int_{K(r / 2, r)}|f(x)|^{2} d x .
$$

Furthermore if $f(x) \in H_{0}^{1}(B(0, r))$, then

$$
\int_{K\left(\rho_{1}, \rho_{2}\right)}|f(x)|^{2} d x \leq C\left[\rho_{2}^{2}-\rho_{1}^{2}\right] \int_{K\left(\rho_{1}, r\right)}|\nabla f|^{2} d x .
$$


We now introduce the following definition.

DeFINITION 2.5. By a weak solution of problem (1.1)-(1.3), we mean a system

$$
\left(\mathcal{S}^{(s)}, \mathcal{F}^{(s)},\left\{\mathcal{F}_{t}^{(s)}\right\}_{0 \leq t \leq T}, \mathcal{P}^{(s)}, W^{(s)}, u^{(s)}\right)
$$

such that for each $s$,

- $\left(\mathcal{S}^{(s)}, \mathcal{F}^{(s)},\left\{\mathcal{F}_{t}^{(s)}\right\}_{0 \leq t \leq T}, \mathcal{P}^{(s)}\right)$ is a probability space, $\left\{\mathcal{F}_{t}^{(s)}\right\}_{0 \leq t \leq T}$ is a filtration on $\left(\mathcal{S}^{(s)}, \mathcal{F}^{(s)}, \mathcal{P}^{(s)}\right)$,

- $W^{(s)}(t)$ is sequence of d-dimensional $\mathcal{F}_{t}^{(s)}$ standard Wiener process,

- $u^{(s)}(t) \in L_{p}\left(\mathcal{S}^{(s)}, L_{\infty}\left(0, T, L_{2}\left(\Omega^{(s)}\right)\right)\right) \cap L_{p / 2}\left(\mathcal{S}^{(s)}, L_{2}\left(0, T, H_{0}^{1}\left(\Omega^{(s)}\right)\right)\right)$, for any $p \in[1, \infty)$,

- for any $\eta \in H_{0}^{1}\left(\Omega^{(s)}\right)$ the integral identity

$$
\begin{aligned}
\left(u^{(s)}(t), \eta\right)=\left(u_{0}, \eta\right)-\int_{0}^{t} \sum_{i=1}^{n}\left(\frac{\partial u^{(s)}(\tau)}{\partial x_{i}}, \frac{\partial \eta}{\partial x_{i}}\right) d \tau \\
+\int_{0}^{t}(f, \eta) d \tau+\sum_{k=1}^{d} \int_{0}^{t}\left(g_{k}, \eta\right) d w_{\tau}^{(s) k}
\end{aligned}
$$

holds for almost all $\omega$ and $t \in[0, T]$.

We assume the following conditions on the data:

$f:(0, T) \times L_{2}\left(\Omega^{(s)}\right) \rightarrow L_{2}\left(\Omega^{(s)}\right)$ is a nonlinear mapping such that

- $f(t, \cdot)$ is measurable for almost every $t$,

- $v \rightarrow f(t, v)$ is continuous from $L_{2}\left(\Omega^{(s)}\right)$ into $L_{2}\left(\Omega^{(s)}\right)$,

$$
\|f(t, v)\|_{L_{2}\left(\Omega^{(s)}\right)} \leq C\left(1+\|v\|_{L_{2}\left(\Omega^{(s)}\right)}\right)
$$

$g_{k}:(0, T) \times L_{2}\left(\Omega^{(s)}\right) \rightarrow L_{2}\left(\Omega^{(s)}\right)$ are nonlinear mappings such that

- $g(t, \cdot)$ is measurable for almost every $t$,

- $v \rightarrow g_{k}(t, v)$ is continuous from $L_{2}\left(\Omega^{(s)}\right)$ into $L_{2}\left(\Omega^{(s)}\right)$,

$$
\begin{aligned}
\left\|g_{k}(t, v)\right\|_{L_{2}\left(\Omega^{(s)}\right)} \leq C\left(1+\|v\|_{L_{2}\left(\Omega^{(s)}\right)}\right) . \\
u_{0}(x) \in L_{2}\left(\Omega^{(s)}\right) .
\end{aligned}
$$

We introduce the space $Z_{\theta}^{(s)}$ (indexed by $\theta>0$ such that $T-\theta>0$ ) of functions $v(t, x)$ defined and measurable on $Q_{T}^{(s)}$ and such that

$$
\begin{gathered}
\sup _{0 \leq t \leq T}\|v\|_{L_{2}\left(\Omega^{(s)}\right)}^{2} \leq C_{1}, \int_{0}^{T}\|v(t)\|_{H_{0}^{1}\left(\Omega^{(s)}\right)}^{2} d t \leq C_{2}, \\
\int_{0}^{T-\theta}\|v(t+\theta)-v(t)\|_{H^{-1}\left(\Omega^{(s)}\right)}^{2} d t \leq C_{3} \theta .
\end{gathered}
$$


We endow $Z_{\theta}^{(s)}$ with the norm

$$
\begin{aligned}
\|v\|_{Z}=\sup _{0 \leq t \leq T} & \|v\|_{L_{2}\left(\Omega^{(s)}\right)}+\left(\int_{0}^{T}\|v(t)\|_{H_{0}^{1}\left(\Omega^{(s)}\right)}^{2} d t\right)^{1 / 2} \\
& +\left(\sup _{\theta>0} \frac{1}{\theta} \int_{0}^{T-\theta}\|v(t+\theta)-v(t)\|_{H^{-1}\left(\Omega^{(s)}\right)}^{2} d t\right)^{1 / 2} .
\end{aligned}
$$

We also consider the space $\mathcal{X}_{p, \theta}^{(s)}(1 \leq p<\infty)$ of random variables $v$ defined on $\mathcal{S}^{(s)} \times$ $Q_{T}^{(s)}$ such that

$$
\begin{gathered}
E^{(s)} \sup _{0 \leq t \leq T}\|v\|_{L_{2}\left(\Omega^{(s)}\right)}^{2 p}<\infty, \quad E^{(s)}\left(\int_{0}^{T}\|v\|_{H_{0}^{1}\left(\Omega^{(s)}\right)}^{2} d t\right)^{p / 2}<\infty, \\
E^{(s)} \int_{0}^{T-\theta}\|v(t+\theta)-v(t)\|_{H^{-1}\left(\Omega^{(s)}\right)}^{2} d t \leq C \theta,
\end{gathered}
$$

where $E^{(s)}$ denotes the mathematical expectation on $\left(\mathcal{S}^{(s)}, \mathcal{F}^{(s)}, \mathcal{P}^{(s)}\right)$. Endowed with the norm

$$
\begin{gathered}
\|v\|_{\mathcal{X}_{p, \theta}^{(s)}}=\left(E^{(s)} \sup _{0 \leq t \leq T}\|v\|_{L_{2}\left(\Omega^{(s)}\right)}^{2 p}\right)^{1 / 2 p}+\left(E^{(s)}\left(\int_{0}^{T}\|v\|_{H_{0}^{1}\left(\Omega^{(s)}\right)}^{2} d t\right)^{p / 2}\right)^{2 / p} \\
+E^{(s)}\left(\sup _{\theta>0} \frac{1}{\theta} \int_{0}^{T-\theta}\|v(t+\theta)-v(t)\|_{H^{-1}\left(\Omega^{(s)}\right)}^{2} d t\right)^{1 / 2}
\end{gathered}
$$

$\mathcal{X}_{p, \theta}^{(s)}$ is a Banach space. We denote by $\tilde{Z}_{\theta}$ and $\tilde{\mathcal{X}}_{p, \theta}$ the corresponding spaces with $\Omega^{(s)}$ replaced by $\Omega$.

The following existence result holds (see, e.g., $[8,40]$ ).

THEOREM 2.6. Under the above conditions, for each $s$, the problem (1.1)-(1.3) has a weak solution

$$
\left(\mathcal{S}^{(s)}, \mathcal{F}^{(s)},\left\{\mathcal{F}_{t}^{(s)}\right\}_{0 \leq t \leq T}, \mathcal{P}^{(s)}, W^{(s)}, u^{(s)}\right)
$$

such that $u^{(s)}$ satisfies

$$
\left\|u^{(s)}\right\|_{\mathcal{X}_{p, \theta}^{(s)}} \leq C
$$

with a constant $C$ independent of $s$, for all $p \geq 1$ and for sufficiently small $\theta>0$.

Note that the equation (2.5) implies that almost surely

$$
u^{(s)}(\cdot) \in C\left(0, T, L_{2}\left(\Omega^{(s)}\right)\right) .
$$

This follows by arguing as in [61, Chap. 3 Par. 3]. Thus the initial condition (1.3) makes sense almost surely. 
Let $\tilde{u}^{(s)}, \tilde{u}_{0}$ be the extension by zero to $Q_{T}$ and to $\Omega$ of the functions $u^{(s)}$ and $u_{0}$, respectively, such that $\mathcal{P}^{(s)}$-almost surely

$$
\begin{gathered}
\tilde{u}^{(s)}(x, t)=u^{(s)}(x, t) \quad \forall(x, t) \in Q_{T}^{(s)}, \tilde{u}^{(s)}(x, t)=0 \forall(x, t) \notin Q_{T}^{(s)}, \\
\tilde{u}_{0}(x)=u_{0}(x) \text { in } \Omega^{(s)} \text { and } \tilde{u}_{0}(x)=0 \text { outside } \Omega^{(s)} .
\end{gathered}
$$

Under the smoothness conditions on $\Omega^{(s)}$ and the fact that $u^{(s)}$ satisfies the Dirichlet boundary conditions such extensions always exist.

These extensions induce extensions $\tilde{f}$ and $\tilde{g}_{k}$ of $f$ and $g_{k}$ from $(0, T) \times L_{2}\left(\Omega^{(s)}\right) \rightarrow$ $L_{2}\left(\Omega^{(s)}\right)$ to $(0, T) \times L_{2}(\Omega) \rightarrow L_{2}(\Omega)$ preserving the inequalities (2.6) and (2.7) with obvious changes of $\Omega^{(s)}$ by $\Omega ; \tilde{u}_{0}(x)$ is bounded in $L_{2}(\Omega)$. Therefore from (2.9) it follows that the function $\tilde{u}^{(s)}$ satisfies the estimate

$$
\left\|\tilde{u}^{(s)}\right\|_{\tilde{\mathcal{X}}_{p, \theta}} \leq C
$$

with the constant $C$ independent of $s$.

Now we consider the set

$$
K=C\left(0, T, \mathbb{R}^{d}\right) \times L_{2}\left(0, T, L_{2}(\Omega)\right)
$$

and $\mathcal{B}(K)$, the $\sigma$-algebra of the Borel sets of $K$. For each $s$, let $\Phi$ be the map

$$
\Phi: \mathcal{S} \rightarrow K: \omega \mapsto\left(W^{(s)}(\omega, \cdot), \tilde{u}^{(s)}(\omega, \cdot)\right) .
$$

For each $s$, we introduce the probability measure $\pi_{s}$ on $(K, \mathcal{B}(K))$ defined by

$$
\pi_{s}(A)=\mathcal{P}^{(s)}\left(\Phi^{-1}(A)\right) \text { for all } A \in \mathcal{B}(K) .
$$

We have the following result.

TheOREM 2.7. The family of probability measures $\left\{\pi_{s}: s \in \mathbb{N}\right\}$ is tight.

The proof of the theorem can be carried out following [8] and [40] (see also [49]). Thus we have, from Prokhorov's compactness result $([47,17])$, that there exists a subsequence $\left\{\pi_{s_{j}}\right\}$ and a measure $\pi$ such that

$$
\pi_{s_{j}} \rightarrow \pi
$$

weakly. By Skorokhod's limit theorem $([57,17])$, there exist a probability space $(\Xi, G, P)$ and random variables $\left(W^{\left(s_{j}\right)}, u^{\left(s_{j}\right)}\right),(W, u)$ on $(\Xi, G, P)$ with values in $K$ such that the probability law of $\left(W^{\left(s_{j}\right)}, u^{\left(s_{j}\right)}\right)$ is $\pi_{s_{j}}$; hence $\left\{W^{\left(s_{j}\right)}\right\}$ is a sequence of $d$-dimensional Wiener processes. Furthermore

$$
\left(W^{\left(s_{j}\right)}, u^{\left(s_{j}\right)}\right) \rightarrow(W, u) \text { in } K, P \text {-a.s. }
$$

and the probability law of $(W, u)$ is $\pi$.

Set

$$
G_{t}=\sigma\{W(\tau), u(\tau)\}_{\tau \in[0, t]}
$$


We show that $W(t)$ is a $G_{t}$-standard Wiener process. For this we use the following characterization of Wiener processes through their characteristic functions (see [25]) which stipulates that for any $m \in \mathbb{N}, 0=t_{0}<t_{1}<\cdots<t_{m}$ and $v_{0}, v_{1}, \ldots, v_{m} \in \mathbb{R}^{d}$,

$$
\begin{aligned}
& E \exp \left\{\sum_{k=1}^{m} i v_{k} \cdot\left[W\left(t_{k}\right)-W\left(t_{k-1}\right)\right]-i v_{0} \cdot W\left(t_{0}\right)\right\} \\
= & \exp \left\{-\frac{1}{2} \sum_{k=1}^{N_{j}}\left|v_{k}\right|^{2}\left(t_{k}-t_{k-1}\right)\right\},
\end{aligned}
$$

where $E$ denotes the mathematical expectation over $(\Xi, G, P)$. (2.12) will follow if we can show that for the conditional characteristic function we have

$$
E\left[\exp \{i v \cdot[W(t+h)-W(t)]\} / G_{t}\right]=\exp \left(-\frac{|v|^{2} h}{2}\right)
$$

for all $h>0$ and any $v \in \mathbb{R}^{d}$. Note that for any given $\sigma$-algebra $G$ and random variables $X$ and $Y$ on a probability space $(\Xi, G, P)$ (it can be any probability space) on which the mathematical expectation is denoted by $E$, if $X$ is $G$-measurable and $E|Y|, E|X Y|<$ $\infty$, then

$$
E(X Y \mid G)=X E(Y \mid G), E E(Y \mid G)=E(Y)
$$

that is

$$
E(X Y)=E(X E(Y \mid G)) .
$$

Using this fact we see that (2.13) will be proved if for any continuous bounded functional $\Lambda_{t}(W(\cdot), u(\cdot))$ on $\Xi$ depending only on the values of $W$ and $u$ on the interval $(0, t)$, we have

$$
\begin{aligned}
& E\left[\exp \{v \cdot[W(t+h)-W(t)]\} \Lambda_{t}(W(\cdot), u(\cdot))\right] \\
= & \exp \left(-\frac{|v|^{2} h}{2}\right) E \Lambda_{t}(W(\cdot),, y(\cdot))
\end{aligned}
$$

Since $\left[W^{\left(s_{j}\right)}(t+h)-W^{\left(s_{j}\right)}(t)\right]$ are independent of $\Lambda_{t}\left(W^{\left(s_{j}\right)}, u^{\left(s_{j}\right)}\right)$ and $W^{\left(s_{j}\right)}$ is a Wiener process,

$$
\begin{aligned}
& E\left[\exp \left\{i v \cdot\left[W^{\left(s_{j}\right)}(t+h)-W^{\left(s_{j}\right)}(t)\right]\right\} \Lambda_{t}\left(W^{\left(s_{j}\right)}, u^{\left(s_{j}\right)}\right)\right] \\
= & E \exp \left\{i v \cdot\left[W^{\left(s_{j}\right)}(t+h)-W^{\left(s_{j}\right)}(t)\right]\right\} E \Lambda_{t}\left(W^{\left(s_{j}\right)}, u^{\left(s_{j}\right)}\right) \\
= & \exp \left(-\frac{|v|^{2} h}{2}\right) E \Lambda_{t}\left(W^{\left(s_{j}\right)}, u^{\left(s_{j}\right)}\right) .
\end{aligned}
$$

In view of (2.11) and the continuity of $\Lambda_{t}$ we can pass to the limit in this equality and get (2.14). The required claim is therefore proved.

A crucial step is to note that the pair $\left(u^{\left(s_{j}\right)}, W^{\left(s_{j}\right)}\right)$ satisfies the problem (1.1)(1.3). We note that this a not a subsequence of $\left(u^{(s)}, W^{(s)}\right)$; they only share at this stage the same law. This fact may seem obvious but in view of the nonlinearity considered in the noise, the proof requires some work; we follow [8] and [49]. 
Since we shall be using $\left(u^{\left(s_{j}\right)}, W^{\left(s_{j}\right)}\right)$ and $\left(u^{(s)}, W^{(s)}\right)$ at the same time, in order to avoid some confusion we temporary denote $u^{\left(s_{j}\right)}$ and $W^{\left(s_{j}\right)}$ by $y^{\left(s_{j}\right)}$ and $B^{\left(s_{j}\right)}$, respectively. We shall revert to the original notation later.

From (1.1)-(1.3), we have

$$
\begin{aligned}
& u^{(s)}(t, x) \\
= & u_{0}(x)+\int_{0}^{t}\left[\Delta u^{(s)}(r, x)+f\left(r, u^{(s)}(r, x)\right)\right] d r+\int_{0}^{t} G\left(r, u^{(s)}(r, x)\right) d W_{r}^{(s)}, \text { in } Q_{T}^{(s)},
\end{aligned}
$$

where

$$
G\left(r, u^{(s)}(r, x)\right) d W^{(s)}=\sum_{k=1}^{d} g_{k}\left(r, u^{(s)}(r, x)\right) d w_{r}^{(s) k}
$$

We set

$$
\Psi_{s}(t)=\Psi\left(u^{(s)}(t), G\left(r, u^{(s)}\right)\right)
$$

where

$$
\begin{aligned}
& \Psi\left(u^{(s)}(t), G\left(r, u^{(s)}\right), W^{(s)}\right) \\
= & u^{(s)}(t, x)-u_{0}(x)-\int_{0}^{t}\left[\Delta u^{(s)}(r, x)+f\left(r, u^{(s)}(r, x)\right)\right] d r \\
& -\int_{0}^{t} G\left(r, u^{(s)}(r, x)\right) d W_{r}^{(s)}
\end{aligned}
$$

and

$$
X_{s}(\omega)=\int_{0}^{T}\left\|\Psi_{s}(t)\right\|_{H^{-1}}^{2} d t
$$

Hence almost surely

$$
X_{s}=0
$$

and

$$
E^{(s)} \frac{X_{s}}{1+X_{s}}=0
$$

since $X_{s} \geq 0$ a.s.

Let

$$
\Psi_{s_{j}}(t)=\Psi\left(y^{\left(s_{j}\right)}(t), G\left(r, y^{\left(s_{j}\right)}\right), B^{\left(s_{j}\right)}\right), \text { in } Q_{T}^{\left(s_{j}\right)},
$$

and

$$
Y_{s_{j}}(\omega)=\int_{0}^{T}\left\|\Psi_{s_{j}}(t)\right\|_{H^{-1}}^{2} d t .
$$

Our claims will be proved if we can show that

$$
E \frac{Y_{s_{j}}}{1+Y_{s_{j}}}=0 .
$$


An obstacle in the realization of this goal is the fact that $X_{s}$ is not a deterministic functional of $u^{(s)}$ and $W^{(s)}$ in view of the presence of the stochastic integral in $X_{s}$. In order to circumvent that difficulty we introduce a regularization of $G$ with respect to $t$, given by

$$
G^{\varepsilon}(y)(t)=\frac{1}{\varepsilon} \int_{0}^{T} \rho\left(-\frac{t-r}{\varepsilon}\right) G(r, y(r)) d r,
$$

where $\rho$ is a mollifier. We have that

$$
E^{(s)} \int_{0}^{T}\left\|G^{\varepsilon}(y)(t)\right\|_{L_{2}\left(\Omega^{(s)}\right)}^{2} d t \leq E^{(s)} \int_{0}^{T}\|G(t, y(t))\|_{L_{2}\left(\Omega^{(s)}\right)}^{2} d t
$$

and

$$
G^{\varepsilon}(y)(\cdot) \rightarrow G(y)(\cdot) \text { in } L_{2}\left(\mathcal{S}^{(s)}, L_{2}\left(0, T, L_{2}\left(\Omega^{(s)}\right)\right)\right),
$$

as $\varepsilon \rightarrow 0$. Next, we set

$$
\begin{aligned}
X_{s, \varepsilon} & =\int_{0}^{T}\left\|\Psi_{s, \varepsilon}(t)\right\|_{H^{-1}}^{2} d t ; \Psi_{s, \varepsilon}(t)=\Psi\left(u^{(s)}(t), G^{\varepsilon}\left(r, u^{(s)}\right), W^{(s)}\right), \\
Y_{s_{j}, \varepsilon} & =\int_{0}^{T}\left\|\Psi_{s_{j}, \varepsilon}(t)\right\|_{H^{-1}}^{2} d t ; \Psi_{s_{j}, \varepsilon}(t)=\Psi\left(u^{\left(s_{j}\right)}(t), G^{\varepsilon}\left(r, u^{\left(s_{j}\right)}\right), B^{\left(s_{j}\right)}\right),
\end{aligned}
$$

and introduce the mapping

$$
\phi_{s, \varepsilon}: C\left(0, T, \mathbf{R}^{d}\right) \times L_{2}\left(0, T, L_{2}\left(\Omega^{(s)}\right)\right) \rightarrow\left(\mathcal{S}^{(s)}, \mathcal{F}^{(s)}, \mathcal{P}^{(s)}\right)
$$

given by

$$
\phi_{s, \varepsilon}\left(W^{(s)}, u^{(s)}\right)=\frac{X_{s, \varepsilon}}{1+X_{s, \varepsilon}} .
$$

Owing to the definition of $X_{s, \varepsilon}$, it is easy to see that $\phi_{s, \varepsilon}$ is bounded and measurable. Similarly, let

$$
\phi_{s_{j}, \varepsilon}\left(B^{\left(s_{j}\right)}, y^{\left(s_{j}\right)}\right)=\frac{Y_{s_{j}, \varepsilon}}{1+Y_{s_{j}, \varepsilon}} .
$$

We use the conclusions of Skorokhod's theorem as follows. Since the laws of $\left(B^{\left(s_{j}\right)}, y^{\left(s_{j}\right)}\right)$ and $\left(W^{\left(s_{j}\right)}, u^{\left(s_{j}\right)}\right)$ are identical,

$$
\begin{aligned}
E \frac{Y_{s_{j}, \varepsilon}}{1+Y_{s_{j}, \varepsilon}} & =E \phi_{s_{j}, \varepsilon}\left(B^{\left(s_{j}\right)}, y^{\left(s_{j}\right)}\right)=\int_{S} \phi_{s_{j}, \varepsilon}(w, x) d \pi_{s_{j}} \\
& =E^{\left(s_{j}\right)} \phi_{s_{j}, \varepsilon}\left(W^{\left(s_{j}\right)}, u^{\left(s_{j}\right)}\right)=E^{\left(s_{j}\right)} \frac{X_{s_{j}, \varepsilon}}{1+X_{s_{j}, \varepsilon}} .
\end{aligned}
$$

But

$$
\begin{aligned}
& E \frac{Y_{s_{j}}}{1+Y_{s_{j}}}-E^{\left(s_{j}\right)} \frac{X_{s_{j}}}{1+X_{s_{j}}} \\
= & E\left(\frac{Y_{s_{j}}}{1+Y_{s_{j}}}-\frac{Y_{s_{j}, \varepsilon}}{1+Y_{s_{j}, \varepsilon}}\right)+E \frac{Y_{s_{j}, \varepsilon}}{1+Y_{s_{j}, \varepsilon}}
\end{aligned}
$$




$$
-E^{\left(s_{j}\right)} \frac{X_{s_{j}, \varepsilon}}{1+X_{s_{j}, \varepsilon}}+E^{\left(s_{j}\right)}\left(\frac{X_{s_{j}, \varepsilon}}{1+X_{s_{j}, \varepsilon}}-\frac{X_{s_{j}}}{1+X_{s_{j}}}\right) .
$$

Hence

$$
\begin{aligned}
E\left|\frac{Y_{s_{j}}}{1+Y_{s_{j}}}-\frac{Y_{s_{j}, \varepsilon}}{1+Y_{s_{j}, \varepsilon}}\right| & =E\left|\frac{Y_{s_{j}}-Y_{s_{j}, \varepsilon}}{\left(1+Y_{s_{j}}\right)\left(1+Y_{s_{j}, \varepsilon}\right)}\right| \leq E\left|Y_{s_{j}}-Y_{s_{j}, \varepsilon}\right| \\
& \leq C\left(E \int_{0}^{T} \| g^{\varepsilon}\left(y^{\left(s_{j}\right)}\right)(t)-\left.g\left(y^{\left(s_{j}\right)}\right)(t)\right|_{L_{2}\left(\Omega^{\left(s_{j}\right)}\right)} ^{2} d t\right)^{1 / 2}
\end{aligned}
$$

and

$$
E^{\left(s_{j}\right)}\left|\frac{X_{s_{j}, \varepsilon}}{1+X_{s_{j}, \varepsilon}}-\frac{X_{s_{j}}}{1+X_{s_{j}}}\right| \leq C\left(E^{\left(s_{j}\right)} \int_{0}^{T}\left\|g^{\varepsilon}\left(u^{\left(s_{j}\right)}\right)(t)-g\left(u^{\left(s_{j}\right)}\right)(t)\right\|_{L_{2}\left(\Omega^{2}\left(s_{j}\right)\right.}^{2} d t\right)^{1 / 2} .
$$

The right-hand sides in these estimates converge to zero as $\varepsilon \rightarrow 0$, in view of (2.18) (reformulated accordingly on the probability space $(\Xi, G, P)$. Combining these relations with (2.19), we get

$$
\| E \frac{Y_{s_{j}}}{1+Y_{s_{j}}}|-| E^{\left(s_{j}\right)} \frac{X_{s_{j}}}{1+X_{s_{j}}}|| \leq\left|E \frac{Y_{s_{j}}}{1+Y_{s_{j}}}-E^{\left(s_{j}\right)} \frac{X_{s_{j}}}{1+X_{s_{j}}}\right| \leq 0 .
$$

It therefore follows that

$$
\left|E \frac{Y_{s_{j}}}{1+Y_{s_{j}}}\right|=\left|E^{\left(s_{j}\right)} \frac{X_{s_{j}}}{1+X_{s_{j}}}\right|=0 .
$$

This proves (2.15) and hence we get

$$
\begin{gathered}
d u^{\left(s_{j}\right)}=\left[\Delta u^{\left(s_{j}\right)}+f\left(t, u^{\left(s_{j}\right)}\right)\right] d t+\sum_{k=1}^{d} g_{k}\left(t, u^{\left(s_{j}\right)}\right) d w_{t}^{\left(s_{j}\right) k}, \text { in } Q_{T}^{\left(s_{j}\right)}, \\
u^{\left(s_{j}\right)}(t, x)=0, \text { on } \partial Q_{T}^{\left(s_{j}\right)}, \\
u^{\left(s_{j}\right)}(0, x)=u_{0}(x), \text { in } \Omega^{\left(s_{j}\right)} .
\end{gathered}
$$

Next we have that the extension of $u^{\left(s_{j}\right)}$ to $Q_{T}$, which we denote again by $u^{\left(s_{j}\right)}$, satisfies the estimate (2.10). As a consequence, the following convergences hold: for any $p \in[1, \infty)$,

$$
\begin{gathered}
u^{\left(s_{j}\right)} \rightarrow u \text { weakly-star in } L_{p}\left(\Xi, L_{\infty}\left(0, T, L_{2}(\Omega)\right)\right), \\
u^{\left(s_{j}\right)} \rightarrow u \text { weakly in } L_{p / 2}\left(\Xi, L_{2}\left(0, T, H_{0}^{1}(\Omega)\right)\right) .
\end{gathered}
$$

Furthermore

$$
u \in L_{p}\left(\Xi, L_{\infty}\left(0, T, L_{2}(\Omega)\right)\right) \cap L_{p / 2}\left(\Xi, L_{2}\left(0, T, H_{0}^{1}(\Omega)\right)\right),
$$

and almost surely

$$
u(\cdot) \in C\left(0, T, L_{2}(\Omega)\right)
$$


Letting $p=4$, we have that

$$
u^{\left(s_{j}\right)} \in L_{4}\left(\Xi, L_{\infty}\left(0, T, L_{2}(\Omega)\right)\right) .
$$

Combining this fact with (2.11) we deduce via Vitali's convergence theorem that

$$
u^{\left(s_{j}\right)} \rightarrow u \text { strongly in } L_{2}\left(\Xi, L_{2}\left(0, T, L_{2}(\Omega)\right)\right) .
$$

Our aim is to determine the initial boundary value problem satisfied by the function $u$ and estimate its closeness to $u^{(s)}$ in suitable topologies finer than the above weak convergence. For this purpose we need some suitable assumptions on the domains $\Omega^{(s)}$.

Let $B(x, \rho)$ denote the ball of radius $\rho$ centered at $x$. Let $d_{i}^{(s)}$ be the radius of the smallest ball containing $F_{i}^{(s)}$ and $x_{i}^{(s)}$ the center of that ball. We denote by $r_{i}^{(s)}$ the distance between $\overline{B\left(x_{i}^{(s)}, d_{i}^{(s)}\right)}$ and the set $\overline{\cup_{i \neq j} B\left(x_{j}^{(s)}, d_{j}^{(s)}\right) \cup \partial \Omega}$; we shall impose some conditions (forthcoming Hypothesis 1 ) which guaranty that the sequence $\left(r_{i}^{(s)}\right)$ is positive and does not converge to zero rapidly, and hence which prevent $B\left(x_{i}^{(s)}, d_{i}^{(s)}\right)$ from crossing $\partial \Omega$.

$B\left(x_{i}^{(s)}, d_{i}^{(s)}\right)$ lies inside $\Omega$ and $\Omega$ is open, so there exists a ball $B\left(x_{i}^{(s)}, a\right)(a>$ $d_{i}^{(s)}$ for sufficiently large $s$ ) inside $\Omega$ which contains $B\left(x_{i}^{(s)}, d_{i}^{(s)}\right)$. We set $B_{i}^{(s)}=$ $B\left(x_{i}^{(s)}, a\right) \backslash F_{i}^{(s)}$. We consider the deterministic functions $v_{i}^{(s)}(x) \in H^{1}\left(B_{i}^{(s)}\right)$ which are solutions of the boundary value problems

$$
\begin{aligned}
\Delta v_{i}^{(s)}(x) & =0 \text { in } B_{i}^{(s)}, \\
v_{i}^{(s)}(x) & =1 \text { on } \partial F_{i}^{(s)}, \\
v_{i}^{(s)}(x) & =0 \text { on } \partial B\left(x_{i}^{(s)}, a\right) .
\end{aligned}
$$

We set $v_{i}^{(s)}(x)=1$ on $F_{i}^{(s)}$ and $v_{i}^{(s)}(x)=0$ outside $B\left(x_{i}^{(s)}, a\right)$. It is well-known that $(2.27)$ is uniquely solvable. In particular $v_{i}^{(s)}(x)$ is the solution of the variational problem

$$
\inf \left\{\int_{B\left(x_{i}^{(s)}, a\right)}\left|\frac{\partial \varphi}{\partial x}\right|^{2} d x: \varphi(x) \in H_{0}^{1}\left(B\left(x_{i}^{(s)}, a\right)\right), \varphi(x)=1 \text { in } F_{i}^{(s)}\right\} .
$$

We note that this quantity is the capacity $\operatorname{Cap}\left(F_{i}^{(s)}\right)$ of the set $F_{i}^{(s)}$ (see e.g. EvansGariepy [23] for a definition), i.e.,

$$
\operatorname{Cap}\left(F_{i}^{(s)}\right)=\int_{B\left(x_{i}^{(s)}, a\right)}\left|\frac{\partial v_{i}^{(s)}}{\partial x}\right|^{2} d x
$$

By the maximum principle (see e.g. [22, Section 2.2.3]) and the extension of $v_{i}^{(s)}(x)$ by 1 in $F_{i}^{(s)}$, we get that

$$
0 \leq v_{i}^{(s)}(x) \leq 1 \text { in } \overline{B\left(x_{i}^{(s)}, a\right) .}
$$


The following pointwise a priori estimates are well-known (see e.g. [30] for proof in the case of higher-order elliptic equations and $[51,53,58]$ for quasilinear elliptic models):

$$
\begin{gathered}
\int_{B\left(x_{i}^{(s)}, a\right)}\left|\frac{\partial v_{i}^{(s)}}{\partial x}\right|^{2} d x \leq C_{1}\left[d_{i}^{(s)}\right]^{n-2}, \\
\left|D^{\alpha} v_{i}^{(s)}(x)\right| \leq C_{2} \frac{\left[d_{i}^{(s)}\right]^{n-2}}{\left|x-x_{i}^{(s)}\right|^{n-2+|\alpha|}} \text { if } d_{i}^{(s)}<\left|x-x_{i}^{(s)}\right|<a,
\end{gathered}
$$

for any $\alpha=\left(\alpha_{1}, \ldots, \alpha_{n}\right)$ with non-negative integer components such that $|\alpha| \leq 1, D^{\alpha}=$ $D_{1}^{\alpha_{1}} \cdots D_{n}^{\alpha_{n}}, D_{i}=\partial / \partial x_{i}$; the constants $C_{1}$ and $C_{2}$ are independent of $s$. Combining the last estimate with the regularity results of the gradient of the solution of Dirichlet problem for elliptic equations (see [32] or [26]), we have

$$
\left\|\frac{\partial v_{i}^{(s)}}{\partial x}\right\|_{L_{\infty}\left(B\left(x_{i}^{(s)}, 1\right)\right)} \leq \frac{C}{d_{i}^{(s)}}
$$

with the constant $C$ independent on $s$. It is easy to check the sharpness of this estimate when $F_{i}^{(s)}$ is a ball.

We impose on the perforated domain $\Omega^{(s)}$ the following conditions. There exist constants $A_{1}$ and $A_{2}$ independent of $s$ such that

H1.

$\mathrm{H} 2$.

$$
d_{i}^{(s)} \leq A_{1} r_{i}^{(s)}, \lim _{s \rightarrow \infty} \max _{1 \leq i \leq I(s)}\left\{r_{i}^{(s)}\right\}=0
$$

$$
\sum_{i=1}^{I(s)} \frac{\left[d_{i}^{(s)}\right]^{2(n-2)}}{\left[r_{i}^{(s)}\right]^{n}} \leq A_{2} .
$$

H3. There exists a bounded function $c(x)$ such that for any open set $G \subset \Omega$,

$$
\lim _{s \rightarrow \infty} \sum_{i \in I_{s}(G)} \operatorname{Cap}\left(F_{i}^{(s)}\right)=\int_{G} c(x) d x
$$

where $I_{s}(G)$ is the set of indices $i \in\{1,2, \ldots, I(s)\}$ such that $F_{i}^{(s)} \subset G$.

The main result of the paper is the following.

THEOREM 2.8. Let the conditions (2.6), (2.7), and (2.8) on the data hold and assume that the hypotheses H1, H2, and H3 are satisfied. Given a sequence of weak solutions

$$
\left(\mathcal{S}^{(s)}, \mathcal{F}^{(s)},\left\{\mathcal{F}_{t}^{(s)}\right\}_{0 \leq t \leq T}, \mathcal{P}^{(s)}, W^{(s)}, u^{(s)}\right)
$$

of problem (1.1)-(1.3), there exist a probability space $\left(\Xi,\left\{G_{t}\right\}_{0 \leq t \leq T}, G, P\right)$ and stochastic processes $\left(W^{\left(s_{j}\right)}, u^{\left(s_{j}\right)}\right),(W, u)\left(W=\left(w_{t}^{1}, \ldots, w_{t}^{d}\right)\right.$ is a d-dimensional Wiener process) on this probability space such that $\left(W^{\left(s_{j}\right)}, u^{\left(s_{j}\right)}\right)$ has the same distribution as 
$\left(W^{(s)}, u^{(s)}\right)$ and converges to $(W, u)$ in the sense of (2.11). Furthermore $u$ satisfies almost surely in the sense of distributions the stochastic initial boundary value problem

$$
\begin{gathered}
d u=[\Delta u-c(x) u+f(t, u)] d t+\sum_{k=1}^{d} g_{k}(t, u) d w_{t}^{k}, \text { in } Q_{T}, \\
u(t, x)=0, \text { on } \partial Q_{T}, \\
u(0, x)=u_{0}(x), \text { in } \Omega,
\end{gathered}
$$

where $\partial Q_{T}=(0, T) \times \partial \Omega$. In addition $u^{\left(s_{j}\right)}$ strongly converges to $u$ in $L_{p}\left(\Xi, L_{p}\left(0, T, W_{p}^{1}(\Omega)\right)\right)$ for all $p \in(1,2)$.

REMark 2.2. The existence of the probability system $\left(\Xi,\left\{G_{t}\right\}_{0 \leq t \leq T}, G, P, W, u\right)$ is a consequence of the Prokhorov and Skorokhod's compactness results as obtained above. Therefore the main issues are the construction of the limit problem (2.35)(2.37) and the strong convergence statement. The latter will follow from a deep asymptotic analysis, undertaken in the next section, centered around an appropriate corrector result for the sequence $u^{\left(s_{j}\right)}$ involving the functions $v_{i}^{(s)}$ and some suitable test functions.

REMARK 2.3. The equation (2.35) contains an additional term of capacity type defined in Hypothesis H3. Explicit expressions for the function $c(x)$ may be obtained for special cases, for instance when the sets $F_{i}^{(s)}$ are balls that are periodically distributed. We refer to [38, Chap.1, pages 55-56], [16, 44] for details. Cioranescu and Murat introduce an abstract version of our conditions, so our geometry is a particular case of theirs.

The next sections of the work will be devoted to the proof of Theorem 2.8.

\section{Corrector result of $u^{(s)}$ with remainder term}

3.1. Formulation of the corrector result. In this section we deal with a corrector result with a remainder term for the sequence $u^{\left(s_{j}\right)}$ in terms of the functions $u, v_{i}^{(s)}(x)$ and appropriate test functions that we shall introduce shortly. We prove that the remainder term converges to zero in suitable topologies, making it possible to justify the corrector formula. We start by introducing the test functions alluded to earlier.

We consider the numbers

$$
\rho_{i}^{(s)}=\max \left\{\left(1+\frac{1}{2 A_{1}}\right) d_{i}^{(s)}, \frac{1}{2 A_{3}}\left[r_{i}^{(s)}\right]^{\frac{n}{n-2}} \ln ^{2} r_{i}^{(s)}\right\},
$$

where $A_{1}$ is the constant from Hypothesis $\mathrm{H} 1$ and

$$
A_{3}=\sup _{0<\tau \leq \text { diam } \Omega}\left\{\tau^{\frac{2}{n-2}} \ln ^{2} \tau\right\} .
$$

By the definition of $\rho_{i}^{(s)}$ and the Hypothesis H1, we see that

$$
\rho_{i}^{(s)} \leq d_{i}^{(s)}+\frac{r_{i}^{(s)}}{2} .
$$


Furthermore, for $i \neq j$ we have that $B\left(x_{i}^{(s)}, d_{i}^{(s)}+\frac{r_{i}^{(s)}}{2}\right) \cap B\left(x_{j}^{(s)}, d_{j}^{(s)}+\frac{r_{j}^{(s)}}{2}\right)=\emptyset$. Let $\theta_{1}$ and $\theta_{2}$ be such that $0<\theta_{2}<\theta_{1}<1$. We consider the functions $\psi_{i}^{(s)} \in C_{o}^{\infty}\left(\mathbf{R}^{n}\right)$, such that $0 \leq \psi_{i}^{(s)}(x) \leq 1, \psi_{i}^{(s)}(x)=0$ for $\left|x-x_{i}^{(s)}\right| \geq \theta_{1} \rho_{i}^{(s)}, \psi_{i}^{(s)}(x)=1$ for $\left|x-x_{i}^{(s)}\right| \leq$ $\theta_{2} \rho_{i}^{(s)}$, and

$$
\left|\frac{\partial \psi_{i}^{(s)}}{\partial x}\right| \leq \frac{C}{\rho_{i}^{(s)}}
$$

with the constant $C$ independent of $s$. We note that $\psi_{i}^{(s)}(x) \psi_{j}^{(s)}(x)=0$ for $i \neq j$. Let us consider the following sets of indices:

$$
\begin{aligned}
& I_{s}^{\prime}=\left\{i=1,2, \ldots, I(s):\left(1+\frac{1}{2 A_{1}}\right) d_{i}^{(s)} \geq \frac{1}{2 A_{3}}\left[r_{i}^{(s)}\right]^{\frac{n}{n-2}} \ln ^{2} r_{i}^{(s)}\right\}, \\
& I_{s}^{\prime \prime}=\left\{i=1,2, \ldots, I(s):\left(1+\frac{1}{2 A_{1}}\right) d_{i}^{(s)}<\frac{1}{2 A_{3}}\left[r_{i}^{(s)}\right]^{\frac{n}{n-2}} \ln ^{2} r_{i}^{(s)}\right\} .
\end{aligned}
$$

It is clear that $I_{s}^{\prime} \cap I_{s}^{\prime \prime}=\emptyset$. We have the following lemma.

Lemma 3.1. If the conditions $H 1$ and H2 are satisfied, then

$$
\begin{aligned}
& \lim _{s \rightarrow \infty} \sum_{i \in I_{s}^{\prime}}\left[d_{i}^{(s)}\right]^{n-2}=0, \\
& \lim _{s \rightarrow \infty} \sum_{i \in I_{s}^{\prime \prime}}\left[\rho_{i}^{(s)}\right]^{n}=0 .
\end{aligned}
$$

Proof. We have

$$
\begin{aligned}
\sum_{i \in I_{s}^{\prime}}\left[d_{i}^{(s)}\right]^{n-2} & =\sum_{i \in I_{s}^{\prime}} \frac{\left[d_{i}^{(s)}\right]^{2(n-2)}}{\left[d_{i}^{(s)}\right]^{n-2}} \\
& \leq C \max _{1 \leq i \leq I(s)}\left\{\frac{1}{\left[\ln r_{i}^{(s)}\right]^{2(n-2)}}\right\} \sum_{i \in I_{s}^{\prime}}\left[\frac{\left[d_{i}^{(s)}\right]^{2(n-2)}}{\left[r_{i}^{(s)}\right]^{n}}\right]
\end{aligned}
$$

By condition H2, (3.2) follows from a passage to the limit as $s \rightarrow \infty$ in the above inequality; here we have made use of the definition of the set $I_{s}^{\prime}$.

For the proof of the relation (3.3), we note that since the balls $B\left(x_{i}^{(s)}, d_{i}^{(s)}+\frac{r_{i}^{(s)}}{2}\right)$, $i=1, \ldots, I(s)$ are pairwise disjoint and $\Omega$ is bounded, it follows that

$$
\sum_{i=1}^{I(s)}\left[r_{i}^{(s)}\right]^{n} \leq C
$$

where $C$ is a constant independent of $s$. We have, by the definition of the set $I_{s}^{\prime \prime}$,

$$
\sum_{i \in I_{s}^{\prime \prime}}\left[\rho_{i}^{(s)}\right]^{n} \leq\left[\frac{1}{2 A_{3}}\right]^{n} \max _{1 \leq i \leq I(s)}\left\{\left[r_{i}^{(s)}\right]^{\frac{2 n}{n-2}} \ln ^{2 n} r_{i}^{(s)}\right\} \sum_{i \in I_{s}^{\prime \prime}}\left[r_{i}^{(s)}\right]^{n} .
$$


A passage to the limit in both sides of this inequality and the relation (3.4) yield (3.3). The lemma is proved.

As a consequence of Lemma 3.1, we have

$$
\sum_{i=1}^{I(s)}\left[\rho_{i}^{(s)}\right]^{n} \leq K_{1} .
$$

This readily follows from (3.3) and the estimate

$$
\sum_{i=1}^{I(s)}\left[d_{i}^{(s)}\right]^{n-2} \leq K_{2} .
$$

The constants $K_{1}$ and $K_{2}$ in these inequalities are independent of $s$. To establish (3.6), we apply Hölder's inequality and get

$$
\begin{aligned}
\sum_{i=1}^{I(s)}\left[d_{i}^{(s)}\right]^{n-2} & =\sum_{i=1}^{I(s)} \frac{\left[d_{i}^{(s)}\right]^{n-2}}{\left[r_{i}^{(s)}\right]^{\frac{n}{2}}}\left[r_{i}^{(s)}\right]^{\frac{n}{2}} \\
& \leq\left\{\sum_{i=1}^{I(s)}\left[\frac{\left[d_{i}^{(s)}\right]^{n-2}}{\left[r_{i}^{(s)}\right]^{\frac{n}{2}}}\right]^{2}\left\{\sum_{i=1}^{\frac{1}{2}}\left[r_{i}^{(s)}\right]^{n}\right\}^{\frac{1}{2}} .\right.
\end{aligned}
$$

Thus, (3.6) is an immediate consequence of condition $\mathrm{H} 2$ and the relation (3.4). Obviously (3.6) implies that $d_{i}^{(s)}$ tends to zero as $s \rightarrow \infty$. Therefore the sets $F_{i}^{(s)}$ vanish as $s \rightarrow \infty$.

Next we proceed to the construction of the corrector formula for $u^{(s)}$. Let $p=4$ in (2.25), so that $u \in L_{4}\left(\Xi, L_{\infty}\left(0, T, L_{2}(\Omega)\right)\right) \cap L_{2}\left(\Xi, L_{2}\left(0, T, H_{0}^{1}(\Omega)\right)\right)$. By Theorem 2.1 there exists a sequence $\left(u_{m}\right)_{m=1,2, \ldots} \in L_{4}\left(\Xi, C_{o}^{\infty}\left(Q_{T}\right)\right)$ approximating $u$ in the sense given there. For simplicity we denote from now on a solution $u^{\left(s_{j}\right)}$ of the problem (2.20)-(2.22) (often referred to as problem (1.1)-(1.3)) as $u^{(s)}$. We look for $u^{(s)}$ in the form

$$
u^{(s)}(t, x)=u(t, x)-H_{1 s}(t, x)-H_{2 s}(t, x)+R_{s}(t, x)
$$

where

$$
\begin{aligned}
& H_{1 s}(\omega, t, x)=\sum_{i \in I_{s}^{\prime}} v_{i}^{(s)}(x) u(\omega, t, x) \psi_{i}^{(s)}(x), \\
& H_{2 s}(\omega, t, x)=\sum_{i \in I_{s}^{\prime \prime}} v_{i}^{(s)}(x) u(\omega, t, x) \psi_{i}^{(s)}(x),
\end{aligned}
$$

and $R_{s}(\omega, t, x)$ is the remainder term.

A surprising fact is that the remainder turns out to be expressed in terms of stochastic integrals as

$$
R_{s}(\omega, t, x)=\sum_{k=1}^{d} \int_{0}^{t} G_{s k}(\omega, \tau, x) d w_{\tau}^{(s) k}
$$


where $G_{s k}(\omega, t, x)$ are some appropriate $G_{t}$-measurable functions such that

$$
E \int_{0}^{t}\left|G_{s k}(\omega, \tau, x)\right|^{2} d \tau<\infty, \text { for } t \in[0, T] .
$$

Investigating the convergence of the functions $H_{k s}(k=1,2)$ and the remainder $R_{s}$ is the main issue to be addressed in the work. A direct study on the functions $H_{k s}$ turns out to be difficult since some needed regularity results such as $L_{\infty}$ boundedness of $u$ are not known. We therefore use the sequence $\left\{u_{m}\right\}$ as an auxiliary tool. Namely we rewrite the functions $H_{k s}(k=1,2)$ as

$$
H_{k s}(\omega, t, x)=H_{k s}^{m \prime}(\omega, t, x)+H_{k s}^{m \prime \prime}(\omega, t, x),
$$

where

$$
\begin{aligned}
& H_{1 s}^{m \prime}(\omega, t, x)=\sum_{i \in I_{s}^{\prime}} v_{i}^{(s)}(x) u_{m}(\omega, t, x) \psi_{i}^{(s)}(x), \\
& H_{1 s}^{m \prime \prime}(\omega, t, x)=\sum_{i \in I_{s}^{\prime}} v_{i}^{(s)}(x)\left[u(\omega, t, x)-u_{m}(\omega, t, x)\right] \psi_{i}^{(s)}(x), \\
& H_{2 s}^{m \prime}(\omega, t, x)=\sum_{i \in I_{s}^{\prime \prime}} v_{i}^{(s)}(x) u_{m}(\omega, t, x) \psi_{i}^{(s)}(x), \\
& H_{2 s}^{m \prime \prime}(\omega, t, x)=\sum_{i \in I_{s}^{\prime \prime}} v_{i}^{(s)}(x)\left[u(\omega, t, x)-u_{m}(\omega, t, x)\right] \psi_{i}^{(s)}(x) .
\end{aligned}
$$

From now on we denote all constants depending only on the data and independent of $s$ and $m$ by $C$, and constants depending on $m$ and independent of $s$ will be denoted by $C_{m}$.

The main result of this section is as follows.

Theorem 3.2. As $s \rightarrow \infty$, under the conditions of Theorem 2.8, we have

$$
\begin{gathered}
H_{1 s} \rightarrow 0 \text { strongly in } L_{2}\left(\Xi, L_{2}\left(0, T, H_{0}^{1}(\Omega)\right)\right) \\
H_{2 s} \rightarrow 0 \text { strongly in } L_{p}\left(\Xi, L_{p}\left(0, T, W_{p}^{1}(\Omega)\right)\right) \text { with } p \in(1,2), \\
H_{2 s} \rightarrow 0 \text { weakly in } L_{2}\left(\Xi, L_{2}\left(0, T, H_{0}^{1}(\Omega)\right)\right) \\
R_{s} \rightarrow 0 \text { strongly in } L_{2}\left(\Xi, L_{2}\left(0, T, H_{0}^{1}(\Omega)\right)\right)
\end{gathered}
$$

We immediately note that the last assertion of Theorem 2.8 is a direct consequence of this theorem.

3.2. Proof of Theorem 3.2. We denote the ball $B\left(x_{i}^{(s)}, \theta_{1} \rho_{i}^{(s)}\right)$ by $D_{i}^{(s)}$. Since $\rho_{i}^{(s)} \rightarrow 0$ as $s \rightarrow \infty$, we can assume that for $s$ sufficiently large, $\theta_{1} \rho_{i}^{(s)}$ is less than 1. Recall that $u_{m} \in L_{4}\left(\Xi, C_{o}^{\infty}\left(Q_{T}\right)\right)$ implies that

$$
\left\|u_{m}\right\|_{L_{4}\left(\Xi, C_{o}^{\infty}\left(Q_{T}\right)\right)}=\left(E \sup _{(t, x) \in Q_{T}}\left|D^{\alpha} u_{m}(\omega, t, x)\right|^{4}\right)^{1 / 4}<\infty .
$$


STEP 1. We prove (3.10), i.e.,

$$
\lim _{s \rightarrow \infty} E \int_{0}^{T}\left\|H_{1 s}\right\|_{H_{0}^{1}(\Omega)}^{2} d t=0 .
$$

We have

$$
\begin{aligned}
E \int_{0}^{T}\left\|H_{1 s}^{m \prime}\right\|_{H_{0}^{1}(\Omega)}^{2} d t & \leq E \sum_{i \in I_{s}^{\prime}} \int_{0}^{T}\left\|\frac{\partial}{\partial x}\left[v_{i}^{(s)}(x) u_{m}(\omega, t, x) \psi_{i}^{(s)}(x)\right]\right\|_{L_{2}(\Omega)}^{2} d t \\
& \leq 2\left(I_{1 s}^{m \prime}+I_{2 s}^{m \prime}+I_{3 s}^{m \prime}\right)
\end{aligned}
$$

where

$$
\begin{aligned}
& I_{1 s}^{m \prime}=E \sum_{i \in I_{s}^{\prime}} \int_{Q_{T}}\left|v_{i}^{(s)}\right|^{2}\left|\psi_{i}^{(s)}\right|^{2}\left|\frac{\partial u_{m}}{\partial x}\right|^{2} d x d t, \\
& I_{2 s}^{m \prime}=E \sum_{i \in I_{s}^{\prime}} \int_{Q_{T}}\left|u_{m}\right|^{2}\left|\psi_{i}^{(s)}\right|^{2}\left|\frac{\partial v_{i}^{(s)}}{\partial x}\right|^{2} d x d t, \\
& I_{3 s}^{m \prime}=E \sum_{i \in I_{s}^{\prime}} \int_{Q_{T}}\left|v_{i}^{(s)}\right|^{2}\left|u_{m}\right|^{2}\left|\frac{\partial \psi_{i}^{(s)}}{\partial x}\right|^{2} d x d t .
\end{aligned}
$$

Using Poincaré's inequality, we have

$$
\begin{aligned}
I_{1 s} & \leq\left(E \sup _{(t, x) \in Q_{T}}\left|\frac{\partial u_{m}}{\partial x}\right|^{2}\right) \sum_{i \in I_{s}^{\prime}} \int_{B\left(x_{i}^{(s)}, 1\right)}\left|v_{i}^{(s)}\right|^{2} d x \\
& \leq C_{m} \sum_{i \in I_{s}^{\prime}} \int_{B\left(x_{i}^{(s)}, 1\right)}\left|\frac{\partial v_{i}^{(s)}}{\partial x}\right|^{2} d x \\
& \leq C_{m} \sum_{i \in I_{s}^{\prime}}\left[d_{i}^{(s)}\right]^{n-2},
\end{aligned}
$$

where we have used inequality (2.29).

Next by (2.29), we have

$$
I_{2 s} \leq C_{m} \sum_{i \in I_{s}^{\prime}}\left[d_{i}^{(s)}\right]^{n-2} .
$$

Thanks to the properties of $\psi_{i}^{(s)}(x)$, the boundedness of $v_{i}^{(s)}(x)$, and the definition of $I_{s}^{\prime}$, we have

$$
I_{3 s} \leq C_{m} \sum_{i \in I_{s}^{\prime}}\left[\rho_{i}^{(s)}\right]^{n-2} \leq C_{m} \sum_{i \in I_{s}^{\prime}}\left[d_{i}^{(s)}\right]^{n-2}
$$

We derive from (3.15), (3.16), (3.17), and (3.18) the inequality

$$
E \int_{0}^{T}\left\|H_{1 s}^{m \prime}\right\|_{H_{0}^{1}(\Omega)}^{2} d t \leq C_{m} \sum_{i \in I_{s}^{\prime}}\left[d_{i}^{(s)}\right]^{n-2} .
$$


Next we have

$$
E \int_{0}^{T}\left\|H_{1 s}^{m \prime \prime}\right\|_{H_{0}^{1}(\Omega)}^{2} d t \leq 2\left(I_{1 s}^{m \prime \prime}+I_{2 s}^{m \prime \prime}+I_{3 s}^{m \prime \prime}\right),
$$

where

$$
\begin{aligned}
& I_{1 s}^{m \prime \prime}=E \sum_{i \in I_{s}^{\prime}} \int_{Q_{T}}\left|v_{i}^{(s)}\right|^{2}\left|\psi_{i}^{(s)}\right|^{2}\left|\frac{\partial\left(u_{m}-u\right)}{\partial x}\right|^{2} d x d t, \\
& I_{2 s}^{m \prime \prime}=E \sum_{i \in I_{s}^{\prime}} \int_{Q_{T}}\left|\psi_{i}^{(s)}\right|^{2}\left|u_{m}-u\right|^{2}\left|\frac{\partial v_{i}^{(s)}}{\partial x}\right|^{2} d x d t \\
& I_{3 s}^{m \prime \prime}=E \sum_{i \in I_{s}^{\prime}} \int_{Q_{T}}\left|v_{i}^{(s)}\right|^{2}\left|u_{m}-u\right|^{2}\left|\frac{\partial \psi_{i}^{(s)}}{\partial x}\right|^{2} d x d t .
\end{aligned}
$$

We have, in view of the boundedness of $v_{i}^{(s)}$ and $\psi_{i}^{(s)}$,

$$
\begin{aligned}
I_{1 s}^{m \prime \prime} & \leq E \sum_{i \in I_{s}^{\prime}} \int_{0}^{T} \int_{B\left(x_{i}^{(s)}, \theta_{1} \rho_{i}^{(s)}\right)}\left|\frac{\partial\left(u_{m}-u\right)}{\partial x}\right|^{2} d x d t \\
& \leq E \int_{0}^{T} \int_{\cup_{i \in I_{s}^{\prime}} B\left(x_{i}^{(s)}, \theta_{1} \rho_{i}^{(s)}\right)}\left|\frac{\partial\left(u_{m}-u\right)}{\partial x}\right|^{2} d x d t \\
& \leq E \int_{Q_{T}}\left|\frac{\partial\left(u_{m}-u\right)}{\partial x}\right|^{2} d x d t=\varepsilon_{1}(m),
\end{aligned}
$$

where $\varepsilon_{1}(m) \rightarrow 0$ as $m \rightarrow \infty$; here we have used the fact that the balls $B\left(x_{i}^{(s)}, \theta_{1} \rho_{i}^{(s)}\right)$ are pairwise disjoint.

The estimation of $I_{2 s}^{m \prime \prime}$ is more involved. First we note that the strong convergence of $u_{m}$ to $u$ in $L_{2}\left(\Xi, L_{2}\left(Q_{T}\right)\right)$ implies that $u_{m}$ converges to $u$ in the $d P \times d t \times d x$ measure. Hence by a theorem of Riesz there exists a subsequence $u_{m_{k}}$ of $u_{m}$ which converges almost everywhere in $\Xi \times Q_{T}$; the whole sequence $u_{m}$ does not converge almost everywhere in general. In view of the necessity of this result, we can consider that throughout the approximating sequence $u_{m}$ is actually replaced by the subsequence $u_{m_{k}}$ which achieves the needed convergence, and we therefore denote $u_{m_{k}}$ as $u_{m}$. Now by Egorov's theorem for any $\varepsilon>0$ there exists a set $A_{\varepsilon}$ in the Borel $\sigma$-algebra of $\Xi \times Q_{T}$ with measure $(d P \times d t \times d x)\left(A_{\varepsilon}\right)<\varepsilon$ and

$$
\lim _{m \rightarrow \infty} \sup _{(\omega, t, x) \in\left(\Xi \times Q_{T}\right) \backslash A_{\varepsilon}}\left|\left(u_{m}-u\right)(\omega, t, x)\right|=0 .
$$

By the above remark for any $\varepsilon>0$, there exists the set $A_{\varepsilon}$ such that

$$
\begin{gathered}
I_{2 s}^{\prime \prime} \leq C E \sup _{(\omega, t, x) \in\left(\Xi \times Q_{T}\right) \backslash A_{\varepsilon}}\left|u_{m}-u\right| \sum_{j=1}^{n}\left[\sum_{i \in I_{s}^{\prime}} \int_{B\left(x_{i}^{(s)}, 1\right)}\left|\frac{\partial v_{i}^{(s)}}{\partial x_{j}}\right|^{2} d x\right] \\
+\sum_{i \in I_{s}^{\prime}} \int_{A_{\varepsilon}}\left|\psi_{i}^{(s)}\right|^{2}\left|u_{m}-u\right|^{2}\left|\frac{\partial v_{i}^{(s)}}{\partial x}\right|^{2} d x d t d P .
\end{gathered}
$$


Using (2.31), we estimate the second term above by

$$
C E\left(\left[\min _{i \in I^{\prime} s}\left[d_{i}^{(s)}\right]^{2}\right]^{-1} \int_{\tilde{A}_{\varepsilon}}\left|u-u_{m}\right|^{2} d x d t\right)
$$

where $\tilde{A}_{\varepsilon}$ is the projection of $A_{\varepsilon}$ on $Q_{T}$. We cover the set $\tilde{A}_{\varepsilon}$ with a finite number of balls $b_{\varepsilon}^{l}, l=1, \ldots, L$, and since $\varepsilon$ is an arbitrary positive number, we choose the radius of $b_{\varepsilon}^{l}$ such that its measure meas $\left[b_{\varepsilon}^{l}\right]$ is equal to $\min _{i \in I_{s}^{\prime}}\left[d_{i}^{(s)}\right]^{2} \max _{i \in I_{s}^{\prime}} d_{i}^{(s)}$. Thus by $(2.29)$,

$$
\begin{aligned}
I_{2 s}^{\prime \prime} \leq C E & \sup _{(\omega, t, x) \in\left(\Xi \times Q_{T}\right) \backslash A_{\varepsilon}}\left|u_{m}-u\right| \sum_{i \in I_{s}^{\prime}}\left[d_{i}^{(s)}\right]^{n-2} \\
& +C \max _{i \in I_{s}^{\prime}} d_{i}^{(s)} \sum_{l=1}^{L} E\left(\frac{1}{\operatorname{meas}\left[b_{\varepsilon}^{l}\right]} \int_{b_{\varepsilon}^{l}}\left|u-u_{m}\right|^{2} d x d t\right)
\end{aligned}
$$

The second factor of the first term in the right-hand side of the above inequality converges to zero as $s \rightarrow \infty$ by (3.2), while the first factor is finite and independent of $s$. Hence the first term vanishes as $s \rightarrow \infty$. As $s \rightarrow \infty$, by the Besicovich-Lebesgue differentiation theorem,

$$
\frac{1}{\min _{i \in I_{s}^{\prime}}\left[d_{i}^{(s)}\right]^{2} \max _{i \in I_{s}^{\prime}} d_{i}^{(s)}} \int_{b_{\varepsilon}^{l}}\left|u-u_{m}\right|^{2} d x d t \rightarrow \mid\left.\left(u-u_{m}\right)\left(\text { center of } b_{\varepsilon}^{l}\right)\right|^{2}
$$

which is finite, while the first factor in the second term in the right side in (3.24) converges to 0 as $s \rightarrow \infty$. Hence we have shown that $I_{2 s}^{\prime \prime}$ vanishes as $s \rightarrow \infty$.

Similar arguments show that

$$
I_{3 s}^{m \prime \prime} \leq C_{m} \varepsilon_{3}(s)
$$

where $\varepsilon_{3}(s) \rightarrow 0$ as $s \rightarrow \infty$. Combining these estimates we deduce from (3.20) that

$$
E \int_{0}^{T}\left\|H_{1 s}^{m \prime \prime}\right\|_{H_{0}^{1}(\Omega)}^{2} d t \leq \varepsilon_{1}(m)+C_{m} \varepsilon(s),
$$

with $\varepsilon(s) \rightarrow 0$ as $s \rightarrow \infty$. From (3.19) and (3.25), we get

$$
E \int_{0}^{T}\left\|H_{1 s}\right\|_{H_{0}^{1}(\Omega)}^{2} d t \leq C_{m}\left[\sum_{i \in I_{s}^{\prime}}\left[d_{i}^{(s)}\right]^{n-2}+\varepsilon(s)\right]+\varepsilon_{1}(m) .
$$

Passing to the limit in both sides of this inequality as $s \rightarrow \infty$, and using (3.2) we get

$$
\lim _{s \rightarrow \infty} E \int_{0}^{T}\left\|H_{1 s}\right\|_{H_{0}^{1}(\Omega)}^{2} d t \leq \varepsilon_{1}(m)
$$

A second passage to the limit as $m \rightarrow \infty$ gives (3.14) and therefore (3.10) holds.

STEP 2. Let us establish (3.11), i.e.,

$$
\lim _{s \rightarrow \infty} E \int_{0}^{T}\left\|H_{2 s}(\omega, t, x)\right\|_{W_{p}^{1}(\Omega)}^{p} d t=0 .
$$


We start with the proof of the relation

$$
E \int_{0}^{T}\left\|H_{2 s}^{m \prime}(\omega, t, x)\right\|_{H_{0}^{1}(\Omega)}^{2} d t<C_{m} .
$$

Combining the boundedness of $u_{m}$ and $v_{i}^{(s)}$ with the properties of $\psi_{i}^{(s)}(x)$, and the inequality (2.29), we get

$$
\begin{aligned}
E \int_{0}^{T}\left\|H_{2 s}^{m \prime}\right\|_{H_{0}^{1}(\Omega)}^{2} d t & \leq E \sum_{i \in I_{s}^{\prime \prime}} \int_{0}^{T}\left\|\frac{\partial}{\partial x}\left(v_{i}^{(s)}(x) u_{m} \psi_{i}^{(s)}(x)\right)\right\|_{L_{2}(\Omega)}^{2} d t \\
& \leq C_{m}\left[\sum_{i \in I_{s}^{\prime \prime}}\left[d_{i}^{(s)}\right]^{n-2}+\sum_{i \in I_{s}^{\prime \prime}}\left[\rho_{i}^{(s)}\right]^{n}\right]
\end{aligned}
$$

This inequality together with (3.5), (3.6) gives (3.27).

Next since $H_{2 s}^{m \prime}(\omega, t, x)=0$ outside $\cup_{i \in I_{s}^{\prime \prime}} D_{i}^{(s)}$, by Hölder's inequality we have

$$
\left\|H_{2 s}^{m \prime}\right\|_{W_{p}^{1}(\Omega)}^{p} \leq C\left\|H_{2 s}^{m \prime}\right\|_{H_{0}^{1}(\Omega)}^{2}\left\{\sum_{i \in I_{s}^{\prime \prime}}\left[\rho_{i}^{(s)}\right]^{n}\right\}^{\frac{2}{p}-1}, p \in(1,2) .
$$

Integrating both sides of this inequality with respect to $t$ and passing to the mathematical expectation we get from (3.27) that

$$
E \int_{0}^{T}\left\|H_{2 s}^{m \prime}(\omega, t, x)\right\|_{W_{p}^{1}(\Omega)}^{p} d t \leq C_{m}\left\{\sum_{i \in I_{s}^{\prime \prime}}\left[\rho_{i}^{(s)}\right]^{n}\right\}^{\frac{2}{p}-1}
$$

Repeating the same arguments used in the estimation of $H_{1 s}^{m \prime \prime}$, it follows that

$$
E \int_{0}^{T}\left\|H_{2 s}^{m \prime \prime}(\omega, t, x)\right\|_{H_{0}^{1}(\Omega)}^{2} d t \leq \varepsilon(m),
$$

where $\varepsilon(m) \rightarrow 0$ as $m \rightarrow \infty$. Thus for $p \in(1,2)$,

$$
E \int_{0}^{T}\left\|H_{2 s}^{m \prime \prime}(\omega, t, x)\right\|_{W_{p}^{1}(\Omega)}^{p} d t \leq C\left(E \int_{0}^{T}\left\|H_{2 s}^{m \prime \prime}(\omega, t, x)\right\|_{H_{0}^{1}(\Omega)}^{2} d t\right)^{p / 2} \leq \varepsilon(m)
$$

Hence

$$
E \int_{0}^{T}\left\|H_{2 s}(\omega, t, x)\right\|_{W_{p}^{1}(\Omega)}^{p} d t \leq C_{m}\left\{\sum_{i \in I_{s}^{\prime \prime}}\left[\rho_{i}^{(s)}\right]^{n}\right\}^{\frac{2}{p}-1}+\varepsilon(m) .
$$

Passing to the limit in this inequality as $s \rightarrow \infty$, and using (3.3) and passing to the limit in the resulting relation, we get

$$
\lim _{s \rightarrow \infty} E \int_{0}^{T}\left\|H_{2 s}(\omega, t, x)\right\|_{W_{p}^{1}(\Omega)}^{p} d t=0 .
$$


This proves (3.11). On the other hand (3.27) and (3.30) imply that $H_{2 s}$ is bounded in $L_{2}\left(\Xi, L_{2}\left(0, T, H_{0}^{1}(\Omega)\right)\right)$, and thus it converges weakly to a function $\xi$ which is equal almost everywhere to 0 in view of (3.11). The statement (3.12) is therefore established.

STEP 3. We proceed to the proof of (3.13). It is the most involved part of the work. In view of (2.24), (3.10), (3.12), and the relation (3.7), we have that $R_{s} \in L_{2}\left(\Xi, L_{2}\left(0, T, H_{0}^{1}(\Omega)\right)\right)$ and

$$
R_{s} \rightarrow 0 \text { weakly in } L_{2}\left(\Xi, L_{2}\left(0, T, H_{0}^{1}(\Omega)\right)\right) .
$$

This does not imply strong convergence of $R_{s}$ in $L_{2}\left(\Xi, L_{2}\left(0, T, L_{2}(\Omega)\right)\right)$ directly. The latter follows from the strong convergence of $H_{2 s}$ to zero in $L_{2}\left(\Xi, L_{2}\left(0, T, L_{2}(\Omega)\right)\right)$, which can be established by rephrasing some parts of the proof of (3.10). This fact, combined with the strong convergence $(2.26)$ of $u^{(s)}$ to $u$ in $L_{2}\left(\Xi, L_{2}\left(0, T, L_{2}(\Omega)\right)\right)$ and (3.10), enables us to claim that

$$
R_{s} \longrightarrow 0 \text { strongly in } L_{2}\left(\Xi, L_{2}\left(0, T, L_{2}(\Omega)\right)\right) .
$$

Let $y_{n}^{(s)}(\omega, t, x)=\zeta_{n}(t) R_{s}(\omega, t, x), n=1,2, \ldots$ be a sequence of random functions in $L_{2}\left(\Xi, L_{2}\left(0, T, H_{0}^{1}(\Omega)\right)\right)$, where $\zeta_{n}(t) \in C_{o}^{\infty}(\mathbf{R}), \zeta_{n}(t)=1$ on $\left[\frac{1}{n}, T-\frac{1}{n}\right]$, and $\zeta_{n}(t)=0$ outside $(0, T)$. We have $y_{n}^{(s)}(t)=0$ outside $(0, T)$ and

$$
\lim _{n \rightarrow \infty} \int_{0}^{T} \int_{\Omega^{(s)}}\left|y_{n}^{(s)}(\omega, t, x)\right|^{2} d t=\int_{0}^{T} \int_{\Omega^{(s)}}\left|R_{s}(\omega, t, x)\right|^{2} d t .
$$

We recall the following formula of integration by parts:

$$
\begin{array}{r}
\int_{0}^{T} \int_{\Omega^{(s)}} y_{n}^{(s)} d u^{(s)} d x=\int_{\Omega^{(s)}} y_{n}^{(s)}(., T, .) u^{(s)}(., T, .) d x-\int_{\Omega^{(s)}} y_{n}^{(s)}(., 0, .) u^{(s)}(., 0, .) d x \\
-\int_{0}^{T} \int_{\Omega^{(s)}} u^{(s)} d y_{n}^{(s)} d x-\int_{0}^{T} \int_{\Omega^{(s)}} d y_{n}^{(s)} d u^{(s)} d x .
\end{array}
$$

Multiplying the equation (1.1) by $y_{n}^{(s)}$ and integrating both sides of the resulting equation over the cylinder $Q_{T}^{(s)}$, we get

$$
\begin{gathered}
-\int_{0}^{T} \int_{\Omega^{(s)}} y_{n}^{(s)} \Delta u^{(s)} d x d t=-\int_{\Omega^{(s)}} \int_{0}^{T} y_{n}^{(s)} d u^{(s)} d x+\int_{0}^{T} \int_{\Omega^{(s)}} y_{n}^{(s)} f\left(t, u^{(s)}\right) d x d t \\
+\sum_{k=1}^{d} \int_{0}^{T} \int_{\Omega^{(s)}} y_{n}^{(s)} g_{k}\left(t, u^{(s)}\right) d x d w_{t}^{k}
\end{gathered}
$$

Further integrating by parts over the integral containing $\Delta u^{(s)}$ and the one containing $y_{n}^{(s)} d u^{(s)}$ (using formula (3.35)), and using the above multiplication rule and the fact that $y_{n}^{(s)}(., 0,)=.y_{n}^{(s)}(., T,)=$.0 , after passage to mathematical expectation we get

$$
\begin{aligned}
& E \sum_{j=1}^{n} \int_{0}^{T} \int_{\Omega^{(s)}} \zeta_{n}(t) \frac{\partial u^{(s)}}{\partial x_{j}} \frac{\partial R_{s}}{\partial x_{j}} d x d t \\
= & E \int_{0}^{T}\left(f^{(s)}, \zeta_{n}(t) R_{s}\right) d t+E \int_{0}^{T}\left(u^{(s)}, R_{s}\right) \frac{\partial \zeta_{n}(t)}{\partial t} d t
\end{aligned}
$$




$$
\begin{aligned}
& +E \sum_{k=1}^{d} \int_{0}^{T} \zeta_{n}(t)\left(g_{k}^{(s)}, R_{s}\right) d w_{t}^{(s) k} \\
& +\sum_{k=1}^{d} \int_{0}^{T} \zeta_{n}(t)\left(u^{(s)}, G_{s k}\right) d w_{t}^{(s) k}+E \sum_{k=1}^{d} \int_{0}^{T} \zeta_{n}(t)\left(g_{k}, G_{s k}\right) d t \\
& \equiv M_{1 s}^{(n)}+M_{2 s}^{(n)}+M_{3 s}^{(n)}+M_{4 s}^{(n)}+M_{5 s}^{(n)} .
\end{aligned}
$$

Taking into account (3.33), we see that the first two terms $M_{1 s}^{(n)}, M_{2 s}^{(n)}$ in the righthand side of (3.36) converge to zero. Let us represent the left-hand side of the equation (3.36) in the form

$$
E \sum_{j=1}^{n} \int_{0}^{T} \int_{\Omega^{(s)}} \zeta_{n}(t) \frac{\partial u^{(s)}}{\partial x_{j}} \frac{\partial R_{s}}{\partial x_{j}} d x d t=I_{1 s}-I_{2 s}-I_{3 s}+I_{4 s}
$$

where

$$
\begin{aligned}
& I_{1 s}=E \sum_{j=1}^{n} \int_{0}^{T} \int_{\Omega^{(s)}} \zeta_{n}(t) \frac{\partial u}{\partial x_{j}} \frac{\partial R_{s}}{\partial x_{j}} d x d t, \\
& I_{2 s}=E \sum_{j=1}^{n} \int_{0}^{T} \int_{\Omega^{(s)}} \zeta_{n}(t) \frac{\partial H_{1 s}}{\partial x_{j}} \frac{\partial R_{s}}{\partial x_{j}} d x d t, \\
& I_{3 s}=E \sum_{j=1}^{n} \int_{0}^{T} \int_{\Omega^{(s)}} \zeta_{n}(t) \frac{\partial H_{2 s}}{\partial x_{j}} \frac{\partial R_{s}}{\partial x_{j}} d x d t, \\
& I_{4 s}=E \sum_{j=1}^{n} \int_{0}^{T} \int_{\Omega^{(s)}} \zeta_{n}(t) \frac{\partial R_{s}}{\partial x_{j}} \frac{\partial R_{s}}{\partial x_{j}} d x d t
\end{aligned}
$$

here we have omitted the index $n$ in the notation of the $I_{j s}$, for simplicity. Thus by convergence of any $I_{j s}$ when $s \rightarrow \infty$, we shall also generally mean that $n \rightarrow \infty$. By (3.10) and (3.32), and the fact that $u \in L_{2}\left(\Xi, L_{2}\left(0, T, H_{0}^{1}(\Omega)\right)\right)$, it follows that $I_{1 s}$ and $I_{2 s}$ converge to zero as $s \rightarrow \infty$.

We proceed next to the estimation of $I_{3 s}$. We note firstly that since $v_{i}^{(s)}(x)$ is a solution of problem (2.27), it satisfies the integral identity

$$
\sum_{j=1}^{n} \int_{B_{i}^{(s)}} \frac{\partial v_{i}^{(s)}}{\partial x_{j}} \frac{\partial \varphi}{\partial x_{j}} d x=0
$$

for all $\varphi(x) \in H_{0}^{1}\left(B_{i}^{(s)}\right)$; recall that $B_{i}^{(s)}=B\left(x_{i}^{(s)}, 1\right) \backslash F_{i}^{(s)}$. Using the definitions of $v_{i}^{(s)}(x)$ and the test functions $\psi_{i}^{(s)}(x)$, we write

$$
I_{3 s}=J_{1 s}+J_{2 s}^{\prime}+J_{3 s}^{\prime}+J_{2 s}^{\prime \prime}+J_{3 s}^{\prime \prime},
$$

where

$$
J_{1 s}=E \sum_{j=1}^{n} \int_{0}^{T} \zeta_{n}(t)\left[\sum_{i \in I_{s}^{\prime \prime}} \int_{D_{i}^{(s)} \backslash F_{i}^{(s)}} \frac{\partial v_{i}^{(s)}}{\partial x_{j}} \frac{\partial}{\partial x_{j}}\left(R_{s} u \psi_{i}^{(s)}\right) d x\right] d t
$$




$$
\begin{aligned}
& J_{2 s}^{\prime}=E \sum_{j=1}^{n} \int_{0}^{T} \zeta_{n}(t)\left[\sum_{i \in I_{s}^{\prime \prime}} \int_{D_{i}^{(s)}} R_{s} \frac{\partial v_{i}^{(s)}}{\partial x_{j}} \frac{\partial}{\partial x_{j}}\left(u_{m} \psi_{i}^{(s)}\right) d x\right] d t, \\
& J_{3 s}^{\prime}=E \sum_{j=1}^{n} \int_{0}^{T} \zeta_{n}(t)\left[\sum_{i \in I_{s}^{\prime \prime}} \int_{D_{i}^{(s)}} v_{i}^{(s)} \frac{\partial R_{s}}{\partial x_{j}} \frac{\partial}{\partial x_{j}}\left(u_{m} \psi_{i}^{(s)}\right) d x\right] d t \\
& J_{2 s}^{\prime \prime}=E \sum_{j=1}^{n} \int_{0}^{T} \zeta_{n}(t)\left[\sum_{i \in I_{s}^{\prime \prime}} \int_{D_{i}^{(s)}} R_{s} \frac{\partial v_{i}^{(s)}}{\partial x_{j}} \frac{\partial}{\partial x_{j}}\left(\left[u-u_{m}\right] \psi_{i}^{(s)}\right) d x\right] d t \\
& J_{3 s}^{\prime \prime}=E \sum_{j=1}^{n} \int_{0}^{T} \zeta_{n}(t)\left[\sum_{i \in I_{s}^{\prime \prime}} \int_{D_{i}^{(s)}} v_{i}^{(s)} \frac{\partial R_{s}}{\partial x_{j}} \frac{\partial}{\partial x_{j}}\left(\left[u-u_{m}\right] \psi_{i}^{(s)}\right) d x\right] d t .
\end{aligned}
$$

For $s$ sufficiently large we may assume that $\theta_{1} \rho_{i}^{(s)}<1$, i.e., $D_{i}^{(s)} \subset B\left(x_{i}^{(s)}, 1\right)$. Thus $\psi_{i}^{(s)}(x)=0$ outside $B\left(x_{i}^{(s)}, 1\right)$. Since $R_{s} u \psi_{i}^{(s)} \in H_{0}^{1}\left(B_{i}^{(s)}\right)$ for almost all $t$ and $\omega$, then taking into account the integral identity (3.38), we get that $J_{1 s}=0$.

In order to estimate $J_{2 s}$ and $J_{3 s}$ we shall need an auxiliary result. For $0<\mu<1$, let $B_{\mu}=\left\{x \in B\left(x_{i}^{(s)}, 1\right): 0 \leq v_{i}^{(s)}(x) \leq \mu\right\}$. We define the function

$$
v_{i \mu}^{(s)}(x)=\left\{\begin{array}{c}
v_{i}^{(s)}(x) \text { if } x \in B_{\mu}, \\
\mu \text { if } x \notin B_{\mu} .
\end{array}\right.
$$

An easy verification shows that the function $\varphi(x)=v_{i \mu}^{(s)}(x)-\mu v_{i}^{(s)}(x)$ is an admissible test function for the integral identity (3.38). Hence substituting it in (3.38) we get

$$
\int_{B\left(x_{i}^{(s)}, 1\right)} \nabla v_{i}^{(s)} \nabla v_{\mu} d x=\mu \int_{B\left(x_{i}^{(s)}, 1\right)}\left|\nabla v_{i}^{(s)}\right|^{2} d x .
$$

The left hand side of this equation is equal to $\int_{B_{\mu}}\left|\nabla v_{i}^{(s)}\right|^{2} d x$. Hence thanks to (3.38) we get the inequality

$$
\int_{B_{\mu}}\left|\nabla v_{i}^{(s)}\right|^{2} d x \leq C \mu\left[d_{i}^{(s)}\right]^{n-2}
$$

By the boundedness of $u_{m}$, we have

$$
J_{2 s}^{\prime} \leq F_{2 s}^{\prime}+F_{2 s}^{\prime \prime},
$$

where

$$
\begin{aligned}
& F_{2 s}^{\prime}=C \sqrt{E \sup _{(t, x) \in Q_{T}}\left|u_{m}\right|^{2}} \sqrt{E\left(\sum_{i \in I_{s}^{\prime \prime}} \int_{0}^{T} \int_{D_{i}^{(s)}}\left|R_{s}\right|\left|\frac{\partial \psi_{i}^{(s)}}{\partial x}\right|\left|\frac{\partial v_{i}^{(s)}}{\partial x}\right| d x d t\right)^{2}}, \\
& F_{2 s}^{\prime \prime}=C \sqrt{E \sup _{(t, x) \in Q_{T}}\left|u_{m}\right|^{2}} \sqrt{E\left(\sum_{i \in I_{s}^{\prime \prime}} \int_{0}^{T} \int_{D_{i}^{(s)}}\left|R_{s}\right|\left|\frac{\partial v_{i}^{(s)}}{\partial x}\right|\left|\psi_{i}^{(s)}\right| d x d t\right)^{2}} .
\end{aligned}
$$


Let us denote $B_{2 i}^{(s)}=D_{i}^{(s)} \backslash B\left(x_{i}^{(s)}, \theta_{2} \rho_{i}^{(s)}\right)$, and also denote the integral in (3.42) by $F_{2}^{\prime}$. Using Hölder's inequality and the definition of $\psi_{i}^{(s)}(x)$, we have

$$
F_{2}^{\prime} \leq\left(\sum_{i \in I_{s}^{\prime \prime}}\left[\rho_{i}^{(s)}\right]^{-2} \int_{B_{2 i}^{(s)}}\left|\frac{\partial v_{i}^{(s)}}{\partial x}\right|^{2} d x\right)^{1 / 2}\left(\sum_{i \in I_{s}^{\prime \prime}} \int_{0}^{T} \int_{D_{i}^{(s)}}\left|R_{s}\right|^{2} d x d t\right)^{1 / 2} .
$$

Let us estimate the integral in the first factor. We set $\mu_{i}^{(s)}=\max _{x \in B_{2 i}^{(s)}}\left|v_{i}^{(s)}(x)\right|$. By (2.30) we have

$$
\mu_{i}^{(s)} \leq C \frac{\left[d_{i}^{(s)}\right]^{n-2}}{\left[\rho_{i}^{(s)}\right]^{n-2}}
$$

Since

$$
\int_{B_{2 i}^{(s)}}\left|\frac{\partial v_{i}^{(s)}}{\partial x}\right|^{2} d x \leq \int_{B_{\mu_{i}^{(s)}}}\left|\frac{\partial v_{i}^{(s)}}{\partial x}\right|^{2} d x
$$

it follows from (3.40) and (3.45) that

$$
\int_{B_{2 i}^{(s)}}\left|\frac{\partial v_{i}^{(s)}}{\partial x}\right|^{2} d x \leq C \mu_{i}^{(s)}\left[d_{i}^{(s)}\right]^{n-2} \leq C \frac{\left[d_{i}^{(s)}\right]^{2(n-2)}}{\left[\rho_{i}^{(s)}\right]^{n-2}} .
$$

This inequality and (3.44) imply

$$
F_{2}^{\prime} \leq \sum_{i \in I_{s}^{\prime \prime}}\left(\frac{\left[d_{i}^{(s)}\right]^{2(n-2)}}{\left[\rho_{i}^{(s)}\right]^{n}}\right)^{1 / 2}\left(\int_{0}^{T} \int_{D_{i}^{(s)}}\left|R_{s}\right|^{2} d x d t\right)^{1 / 2} .
$$

Let us estimate the integral over $D_{i}^{(s)}$ in the right-hand side of (3.46). As noted at the beginning of Section $3, \theta_{1} \rho_{i}^{(s)} \leq \rho_{i}^{(s)} \leq \frac{d_{i}^{(s)}}{2}+r_{i}^{(s)}$. Define $\sigma_{i}^{(s)}=\frac{d_{i}^{(s)}}{2}+r_{i}^{(s)}$. By inequality (2.3) in Lemma 2.4, we have

$$
\begin{aligned}
\int_{D_{i}^{(s)}}\left|R_{s}\right|^{2} d x \leq C\left(\theta_{1} \rho_{i}^{(s)}\right)^{2} \int_{B\left(x_{i}^{(s)}, \sigma_{i}^{(s)}\right)}\left|\frac{\partial R_{s}}{\partial x}\right|^{2} d x \\
+C \frac{\left[\theta_{1} \rho_{i}^{(s)}\right]^{n}}{\left[\sigma_{i}^{(s)}\right]^{n}} \int_{B\left(x_{i}^{(s)}, \sigma_{i}^{(s)}\right)}\left|R_{s}\right|^{2} d x .
\end{aligned}
$$

This inequality and (3.46) give

$$
F_{2}^{\prime} \leq C \sum_{i \in I_{s}^{\prime \prime}}\left(\frac{\left[d_{i}^{(s)}\right]^{2(n-2)}}{\left[\rho_{i}^{(s)}\right]^{n}}\right)^{1 / 2}\left(\int_{0}^{T} \int_{B\left(x_{i}^{(s)}, \sigma_{i}^{(s)}\right)}\left|R_{s}\right|^{2} d x d t\right)^{1 / 2}
$$




$$
+C \sum_{i \in I_{s}^{\prime \prime}}\left(\frac{\left[d_{i}^{(s)}\right]^{2(n-2)}}{\left[\rho_{i}^{(s)}\right]^{n-2}}\right)^{1 / 2}\left(\int_{0}^{T} \int_{B\left(x_{i}^{(s)}, \sigma_{i}^{(s)}\right)}\left|\frac{\partial R_{s}}{\partial x}\right|^{2} d x d t\right)^{1 / 2} .
$$

The balls $B\left(x_{i}^{(s)}, \sigma_{i}^{(s)}\right)$ do not intersect, thus by the definition of $I_{s}^{\prime \prime}$ and (3.42) we get that

$$
\begin{aligned}
& F_{2 s}^{\prime} \leq C_{m} \sqrt{\left(\sum_{i \in I_{s}^{\prime \prime}} \frac{\left[d_{i}^{(s)}\right]^{2(n-2)}}{\left[\rho_{i}^{(s)}\right]^{n}}\right) E\left(\int_{Q_{T}}\left|R_{s}\right|^{2} d x d t\right)} \\
&+C_{m} \sqrt{\sup \left\{\frac{1}{\left[\ln r_{i}^{(s)}\right]^{2(n-2)}}\right\}\left(\sum_{i \in I_{s}^{\prime \prime}} \frac{\left[d_{i}^{(s)}\right]^{2(n-2)}}{\left[r_{i}^{(s)}\right]^{n}}\right)^{1 / 2} E\left(\int_{Q_{T}}\left|\frac{\partial R_{s}}{\partial x}\right|^{2} d x d t\right) .} .
\end{aligned}
$$

The first term in the right-hand side converges to zero thanks to (2.33) and (3.33). The two last factors in the second term are bounded for similar reasons, while the first factor converges to zero. Hence we get that $\lim _{s \rightarrow \infty} F_{2 s}^{\prime}=0$.

Next, by Hölder's inequality we have

$$
F_{2 s}^{\prime \prime} \leq C_{m}\left(E \int_{Q_{T}}\left|R_{s}\right|^{2} d x d t\right)^{1 / 2}\left(\sum_{i \in I_{s}^{\prime \prime}}\left[d_{i}^{(s)}\right]^{n-2}\right)^{1 / 2} .
$$

(3.6) and (3.33) imply that $\lim _{s \rightarrow \infty} F_{2 s}^{\prime \prime}=0$. Hence we have proved that

$$
\lim _{s \rightarrow \infty} J_{2 s}^{\prime}=0 .
$$

Similar arguments show that

$$
\lim _{s \rightarrow \infty} J_{3 s}^{\prime}=0 .
$$

Next we prove that $J_{2 s}^{\prime \prime}$ and $J_{3 s}^{\prime \prime}$ converge to zero as $s \rightarrow \infty$. By Egorov's theorem, for any $\varepsilon>0$ there exists a set $A_{\varepsilon}$ in the Borel $\sigma$-algebra of $\Xi \times Q_{T}$ with measure $(d P \times d t \times d x)\left(A_{\varepsilon}\right)<\varepsilon$ and

$$
\lim _{m \rightarrow \infty} \sup _{(\omega, t, x) \in\left(\Xi \times Q_{T}\right) \backslash A_{\varepsilon}}\left|\left(u_{m}-u\right)(\omega, t, x)\right|=0 .
$$

We have $J_{2 s}^{\prime \prime} \leq G_{2}^{\prime}+G_{2}^{\prime \prime}$, where

$$
\begin{aligned}
& G_{2}^{\prime}=C E \sum_{j=1}^{n} \int_{0}^{T}\left[\sum_{i \in I_{s}^{\prime \prime}} \int_{D_{i}^{(s)}}\left|R_{s}\left[u-u_{m}\right] \frac{\partial v_{i}^{(s)}}{\partial x_{j}} \frac{\partial \psi_{i}^{(s)}}{\partial x_{j}}\right| d x\right] d t \\
& G_{2}^{\prime \prime} \leq C E \sum_{j=1}^{n} \int_{0}^{T}\left[\sum_{i \in I_{s}^{\prime \prime}} \int_{D_{i}^{(s)}}\left|R_{s} \psi_{i}^{(s)} \frac{\partial v_{i}^{(s)}}{\partial x_{j}} \frac{\partial\left[u-u_{m}\right]}{\partial x_{j}}\right| d x\right] d t .
\end{aligned}
$$


Thus

$$
\begin{gathered}
G_{2}^{\prime} \leq C E \sup _{(\omega, t, x) \in\left(\Xi \times Q_{T}\right) \backslash A_{\varepsilon}}\left|u_{m}-u\right| \sum_{j=1}^{n} \int_{0}^{T}\left[\sum_{i \in I_{s}^{\prime \prime}} \int_{D_{i}^{(s)}}\left|R_{s} \frac{\partial v_{i}^{(s)}}{\partial x_{j}} \frac{\partial \psi_{i}^{(s)}}{\partial x_{j}}\right| d x\right] d t \\
+C \sum_{j=1}^{n} \int_{A_{\varepsilon}}\left|R_{s}\left[u-u_{m}\right] \frac{\partial v_{i}^{(s)}}{\partial x_{j}} \frac{\partial \psi_{i}^{(s)}}{\partial x_{j}}\right| d x d t d P .
\end{gathered}
$$

The second term is estimated above by

$$
C\left(E \int_{Q_{T}}\left|R_{s}\right|^{2} d x d t\right)^{1 / 2} E\left(\left[\min _{i \in I_{s}^{\prime \prime}}\left[\rho_{i}^{(s)}\right]^{2}\right]^{-1} \int_{\tilde{A}_{\varepsilon}}\left|u-u_{m}\right|^{2} d x d t\right)^{1 / 2}
$$

where $\tilde{A}_{\varepsilon}$ is the projection of $A_{\varepsilon}$ on $Q_{T}$. We cover the set $\tilde{A}_{\varepsilon}$ with a finite number of balls $b_{\varepsilon}^{l}, l=1, \ldots, L$, and since $\varepsilon$ is an arbitrary positive number, we choose the radius of $b_{\varepsilon}^{l}$ such that its volume is equal to $\min _{i \in I_{s}^{\prime \prime}}\left[\rho_{i}^{(s)}\right]^{2}$. Thus

$$
\begin{array}{r}
G_{2}^{\prime} \leq C E \sup _{(\omega, t, x) \in\left(\Xi \times Q_{T}\right) \backslash A_{\varepsilon}}\left|u_{m}-u\right| \sum_{j=1}^{n} \int_{0}^{T}\left[\sum_{i \in I_{s}^{\prime \prime}} \int_{D_{i}^{(s)}}\left|R_{s} \frac{\partial v_{i}^{(s)}}{\partial x_{j}} \frac{\partial \psi_{i}^{(s)}}{\partial x_{j}}\right| d x\right] d t \\
+C \sqrt{E \int_{Q_{T}}\left|R_{s}\right|^{2} d x d t E} \sqrt{\sum_{l=1}^{L}\left[\min _{i \in I_{s}^{\prime \prime}}\left[\rho_{i}^{(s)}\right]^{2}\right]^{-1} \int_{b_{\varepsilon}^{l}}\left|u-u_{m}\right|^{2} d x d t .}
\end{array}
$$

The second factor of the first term in the right-hand side of the above inequality converges to zero as $s \rightarrow \infty$ (see the investigation of the term $F_{2 s}^{\prime}$ above), while the first factor is finite and independent of $s$. Hence the first term vanishes as $s \rightarrow \infty$. As $s \rightarrow \infty$, by the Besicovich-Lebesgue differentiation theorem,

$$
\frac{1}{\operatorname{meas}\left[b_{\varepsilon}^{l}\right]} \int_{b_{\varepsilon}^{l}}\left|u-u_{m}\right|^{2} d x d t \rightarrow\left|u-u_{m}\right|^{2}\left(\text { center of } b_{\varepsilon}^{l}\right)
$$

which is finite, while the first factor in the second term in the right side in (3.52) converges to 0 as $s \rightarrow \infty$. Hence we have shown that $G_{2}^{\prime}$ vanishes as $s \rightarrow \infty$. Similarly $G_{2}^{\prime \prime} \rightarrow 0$ as $s \rightarrow \infty$. Thus $J_{2 s}^{\prime \prime} \rightarrow 0$ as $s \rightarrow \infty$. Analogously $J_{3 s}^{\prime \prime} \rightarrow 0$ as $s \rightarrow \infty$. Combining all the results of convergence obtained, we get that $I_{3 s}$ (see (3.39)) converges to zero as $s \rightarrow \infty$.

Therefore taking into account that the first three terms in the right-hand side of (3.37) and the first term in the right-hand side of (3.36) vanish at the limit, we have

$$
E \sum_{i=1}^{n} \int_{0}^{T} \int_{\Omega^{(s)}} \zeta_{n}(t)\left|\frac{\partial R_{s}}{\partial x}\right|^{2} d x d t=\varsigma_{s}^{(n)}+M_{3 s}^{(n)}+M_{4 s}^{(n)}+M_{5 s}^{(n)}
$$

where $\varsigma_{s}^{(n)} \rightarrow 0$, as $s, n \rightarrow \infty$.

We proceed to the estimation of the stochastic integral

$$
M_{3 s}^{(n)}=E \sum_{k=1}^{d} \int_{0}^{T} \zeta_{n}(t)\left(g_{k}, R_{s}\right) d w_{t}^{k} .
$$


We have, by the Martingale inequality,

$$
\begin{aligned}
E \sum_{k=1}^{d} \int_{0}^{T} \zeta_{n}(t)\left(g_{k}, R_{s}\right) d w_{t}^{(s) k} & \leq \sum_{k=1}^{d} E\left|\int_{0}^{T}\left(g_{k}, R_{s}\right) d w_{t}^{(s) k}\right| \\
& \leq C \sum_{k=1}^{d} E\left(\int_{0}^{T}\left(g_{k}, R_{s}\right)^{2} d t\right)^{1 / 2} .
\end{aligned}
$$

In view of the condition (2.7) on $g_{k}$, we have

$$
\begin{aligned}
& E \sum_{k=1}^{d} \int_{0}^{T} \zeta_{n}(t)\left(g_{k}, R_{s}\right) d w_{t}^{(s) k} \\
\leq & C\left[E \sup _{0 \leq t \leq T}\left(1+\left\|u^{(s)}\right\|_{L_{2}(\Omega)}^{2}\right)\right]^{1 / 2}\left[E\left(\int_{Q_{T}}\left|R_{s}\right|^{2} d x d t\right)\right]^{1 / 2} .
\end{aligned}
$$

The first factor in the right-hand is bounded and the second vanish at the limit by (3.33). Hence $\lim _{s, n \rightarrow \infty} M_{3 s}^{(n)}=0$.

In order to estimate the last two stochastic integrals $M_{4 s}^{(n)}$ and $M_{5 s}^{(n)}$ we need the relation

$$
\lim _{s \rightarrow \infty, n \rightarrow \infty} E \int_{0}^{T} \int_{\Omega^{(s)}}\left|G_{s k}^{(n)}(\omega, t, x)\right|^{2} d t=0
$$

the validity of which we now establish. From the definition of $y_{n}^{(s)}$, we have by (3.34) and (3.33) that

$$
\lim _{s \rightarrow \infty, n \rightarrow \infty} E \int_{0}^{T} \int_{\Omega^{(s)}}\left|y_{n}^{(s)}(\omega, t, x)\right|^{2} d x d t=0 .
$$

But since (see e.g. [42, page 29])

$$
E\left|\int_{0}^{T}\left(g_{k}, R_{s}\right) d w_{t}^{(s) k}\right|^{2}=E \int_{0}^{T}\left|\left(g_{k}, R_{s}\right)\right|^{2} d t
$$

and the Wiener processes $w_{t}^{k}$ are independent, we have

$$
E \int_{0}^{T} \int_{\Omega^{(s)}}\left[\sum_{k=1}^{d} \int_{0}^{\tau} G_{s k}(x, \tau, \omega) d w_{\tau}^{(s) k}\right]^{2}=E \int_{0}^{T} \int_{\Omega^{(s)}} \int_{0}^{\tau}\left[G_{s k}(x, \tau, \omega)\right]^{2} d \tau d x d t .
$$

Since

$$
E \int_{0}^{T} \int_{\Omega^{(s)}}\left|y_{n}^{(s)}(\omega, t, x)\right|^{2} d x d t=E \int_{0}^{T} \int_{\Omega^{(s)}}\left[\zeta_{n}(t)\right]^{2}\left[\sum_{k=1}^{d} \int_{0}^{\tau} G_{s k}(x, \tau, \omega) d w_{\tau}^{(s) k}\right]^{2},
$$

(3.55) and (3.57) imply that

$$
\lim _{s \rightarrow \infty, n \rightarrow \infty} E \int_{0}^{T} \int_{\Omega^{(s)}} \int_{0}^{t}\left[G_{s k}^{(n)}(x, \tau, \omega)\right]^{2} d \tau d x d t=0 .
$$


Combining this relation with Fubini's theorem and the non-negativity of the integrand, we get (3.54). Arguing as in the estimation of $M_{3 s}^{(n)}$ and taking into account (3.54), we easily show that the integrals $M_{4 s}^{(n)}$ and $M_{5 s}^{(n)}$ in (3.53) converge to zero. Thus passing to the limit in both sides of (3.53) as $n, s \rightarrow \infty$, and using the definition of $\zeta_{n}(t)$, we conclude that

$$
\lim _{s \rightarrow \infty} E \int_{Q_{T}}\left|\frac{\partial R_{s}}{\partial x}\right|^{2} d x d t=\lim _{s \rightarrow \infty} \lim _{n \rightarrow \infty} E \int_{Q_{T}} \zeta_{n}(t)\left|\frac{\partial R_{s}}{\partial x}\right|^{2} d x d t=0 .
$$

The relation (3.13) is proved. This completes the proof of Theorem 3.2.

\section{Derivation of the limit problem}

In this section we construct the limit problem (2.35)-(2.37) satisfied by the function $u(\omega, t, x)$. Let $h(x)$ be an arbitrary function in $C_{o}^{\infty}(\Omega)$. We consider the sequence of functions

$$
h_{s}(x)=h(x)-\sum_{i=1}^{I(s)} h(x) v_{i}^{(s)}(x) \psi_{i}^{(s)}(x), s=1,2, \ldots
$$

where $\psi_{i}^{(s)}(x)$ are the test functions introduced in the previous section and the functions $v_{i}^{(s)}(x)$ are solutions of the problem (2.27). It is clear that $h_{s}(x) \in H_{0}^{1}\left(\Omega^{(s)}\right)$. Let us denote the second term in $(4.1)$ by $G_{s}(x)$. Arguing as in the proof of Theorem 3.2 (see Steps 1 and 2), we see that

$$
h_{s}(x) \rightarrow 0 \text {, weakly in } H_{0}^{1}(\Omega) \text { (strongly in } L_{2}(\Omega) \text { ), }
$$

and

$$
h_{s}(x) \rightarrow 0 \text { strongly in } \stackrel{o}{W_{p}^{1}}(\Omega), p \in(1,2) .
$$

Substituting $\eta(x)=g_{s}(x)$ in the integral identity (2.5), we get

$$
\begin{aligned}
& \sum_{i=1}^{n} \int_{0}^{T} \int_{\Omega^{(s)}} \frac{\partial u^{(s)}}{\partial x_{i}} \frac{\partial h_{s}}{\partial x_{i}} d x d t \\
= & -\int_{\Omega^{(s)}} u^{(s)}(\omega, T, x) h_{s}(x) d x+\int_{\Omega^{(s)}} u_{0}(x) h_{s}(x) d x \\
& +\int_{0}^{T} \int_{\Omega^{(s)}} f\left(t, u^{(s)}\right) h_{s}(x) d x d t+\sum_{k=1}^{d} \int_{0}^{T} \int_{\Omega^{(s)}} h_{s} g_{k}\left(t, u^{(s)}\right) d x d w_{t}^{(s) k} .
\end{aligned}
$$

By (4.2) and the strong convergence $(2.26)$ of $u^{(s)}$, it readily follows that

$$
\begin{aligned}
& E\left[\int_{\Omega^{(s)}} u_{0}(x, \omega) h_{s}(x) d x-\int_{\Omega^{(s)}} u^{(s)}(x, T, \omega) h_{s}(x) d x\right] \\
\rightarrow & E\left[\int_{\Omega} u_{0}(x, \omega) h(x) d x-\int_{\Omega} u(x, T, \omega) h(x) d x\right] .
\end{aligned}
$$

The convergence (2.26) implies, modulo extraction of a suitable subsequence, that for almost every $(\omega, t)$ with respect to the measure $d P \times d t$,

$$
u^{(s)} \rightarrow u \text { strongly in } L_{2}(\Omega)
$$


Combining this with the boundedness of $u^{(s)}$ in $L_{4}\left(\Xi, L_{2}\left(0, T, L_{2}(\Omega)\right)\right)$ and the continuity of $f(t, v)$ in $v$ enables us to appeal to Vitali's convergence theorem to claim that

$$
f\left(t, u^{(s)}\right) \rightarrow f(t, u) \text { in } L_{2}\left(\Xi, L_{2}\left(0, T, L_{2}(\Omega)\right)\right) .
$$

This fact together with (4.2) implies

$$
E \int_{0}^{T} \int_{\Omega} f\left(t, u^{(s)}\right) h_{s}(x) d x d t \rightarrow E \int_{0}^{T} \int_{\Omega} f(t, u) h(x) d x d t .
$$

Similarly, owing to the conditions on $g_{k}$,

$$
g_{k}\left(\cdot, u^{(s)}(\cdot)\right) \rightarrow g_{k}(\cdot, u(\cdot)) \text { in } L_{2}\left(\Xi, L_{2}\left(0, T, L_{2}(\Omega)\right)\right) .
$$

The convergence

$$
E \sum_{k=1}^{d} \int_{0}^{T} \int_{\Omega^{(s)}} h_{s} g_{k}\left(t, u^{(s)}\right) d x d w_{t}^{(s) k} \rightarrow E \sum_{k=1}^{d} \int_{0}^{T} \int_{\Omega} h g_{k}(t, u) d x d w_{t}^{k}
$$

is more involved; it will follow from

$$
\int_{0}^{t} g_{k}\left(r, u^{(s)}(r)\right) d w_{r}^{(s) k} \rightarrow \int_{0}^{t} g_{k}(r, u(r)) d w_{r}^{k} \text { weakly in } L_{2}\left(\Xi, L_{2}(\Omega)\right),
$$

for almost all $t \in[0, T]$. The difficulty here lies in the coupling of the nonlinearity of $g_{k}$ with the presence of a varying Wiener process. To prove (4.8) we introduce the regularization

$$
g_{k}^{\varepsilon}(u)(q)=\frac{1}{\varepsilon} \int_{0}^{t} \rho\left(-\frac{q-r}{\varepsilon}\right) g_{k}(r, u(r)) d r
$$

where $\rho$ is a mollifier and $u \in L_{2}\left(\Xi, L_{2}\left(Q_{T}\right)\right)$. We have that

$$
E \int_{0}^{T}\left\|g_{k}^{\varepsilon}(u)(t)\right\|_{L_{2}(\Omega)}^{2} d t \leq E \int_{0}^{T}\left\|g_{k}(t, u(t))\right\|_{L_{2}(\Omega)}^{2} d t
$$

and

$$
g_{k}^{\varepsilon}(t, u) \rightarrow g_{k}(t, u) \text { strongly in } L_{2}\left(\Xi, L_{2}(\Omega)\right) .
$$

Also, for $s$ fixed,

$$
g_{k}^{\varepsilon}\left(t, u^{(s)}\right) \rightarrow g_{k}\left(t, u^{(s)}\right) \text { in } L_{2}\left(\Xi, L_{2}(\Omega)\right) .
$$

From the definition of $g_{k}^{\varepsilon}$, we have

$$
\begin{aligned}
& \int_{0}^{t}\left\|g_{k}^{\varepsilon}\left(u^{(s)}\right)(r)-g_{k}^{\varepsilon}(u)(r)\right\|_{L_{2}(\Omega)}^{2} d r \\
\leq & \int_{0}^{t}\left\|g_{k}\left(u^{(s)}\right)(r)-g_{k}(u)(r)\right\|_{L_{2}(\Omega)}^{2} d r .
\end{aligned}
$$


Next, by integration by parts we have

$$
\int_{0}^{t} g_{k}^{\varepsilon}\left(u^{(s)}\right)(r) d w_{r}^{(s) k}=g_{k}^{\varepsilon}\left(u^{(s)}\right)(t) w_{t}^{(s) k}-\int_{0}^{t} g_{k}^{\varepsilon \prime}\left(u^{(s)}\right)(r) w_{r}^{(s) k} d r .
$$

In view of (2.11) and (4.6), passing to the limit as $s \rightarrow \infty$ in this relation we get

$$
\int_{0}^{t} g_{k}^{\varepsilon}\left(u^{(s)}\right)(r) d w_{r}^{(s) k} \rightarrow g_{k}^{\varepsilon}(u)(t) w_{t}^{k}-\int_{0}^{t} g_{k}^{\varepsilon \prime}(u)(r) w_{r}^{k} d r
$$

pointwise for almost all $\omega, x$; the right hand side is equal to $\int_{0}^{t} g_{k}^{\varepsilon}(u)(r) d w_{r}^{k}$. Since

$$
E\left|\int_{0}^{t} \int_{\Omega} g_{k}^{\varepsilon}\left(u^{(s)}\right)(r) d x d w_{r}^{(s) k}\right|^{2}=E \int_{0}^{t}\left\|g_{k}^{\varepsilon}\left(u^{(s)}\right)(r)\right\|_{L_{2}(\Omega)}^{2} d r \leq C
$$

it follows from Remark 2.1 that

$$
\int_{0}^{t} g_{k}^{\varepsilon}\left(u^{(s)}\right)(r) d w_{r}^{(s) k} \rightarrow \int_{0}^{t} g_{k}^{\varepsilon}(u)(r) d w_{r}^{k} \text { weakly in } L_{2}\left(\Xi, L_{2}(\Omega)\right),
$$

that is, for all $\phi \in L_{2}\left(\Xi, L_{2}(\Omega)\right)$,

$$
E\left(\phi, \int_{0}^{t} g_{k}^{\varepsilon}\left(u^{(s)}\right)(r) d w_{r}^{(s) k}\right) \rightarrow E\left(\phi, \int_{0}^{t} g_{k}^{\varepsilon}(u)(r) d w_{r}^{k}\right) .
$$

Also

$$
E\left|\int_{0}^{t} \int_{\Omega} g_{k}\left(u^{(s)}\right)(r) d w_{r}^{(s) k}\right|^{2}=E \int_{0}^{t}\left\|g_{k}\left(u^{(s)}\right)(r)\right\|_{L_{2}(\Omega)}^{2} d r \leq C .
$$

Thus there exists $\eta \in L_{2}\left(\Xi, L_{2}(\Omega)\right)$ such that for all $\phi \in L_{2}\left(\Xi, L_{2}(\Omega)\right)$,

$$
E\left(\phi, g_{k}\left(u^{(s)}\right)(r) d w_{r}^{(s) k}\right) \rightarrow E(\phi, \eta) .
$$

We show that

$$
\eta=\int_{0}^{t} g(u)(r) d w_{r}^{k}
$$

We have

$$
\begin{aligned}
& E\left(\phi, \int_{0}^{t} g_{k}\left(u^{(s)}\right)(r) d w_{r}^{(s) k}\right)-E\left(\phi, \int_{0}^{t} g(u)(r) d w_{r}^{k}\right) \\
= & I_{1}+I_{2}+I_{3}
\end{aligned}
$$

where

$$
\begin{aligned}
& I_{1}=E\left(\phi, \int_{0}^{t}\left(g\left(u^{(s)}\right)(r)-g^{\varepsilon}\left(u^{(s)}\right)(r)\right) d w_{r}^{(s) k}\right) \\
& I_{2}=E\left(\phi, \int_{0}^{t} g^{\varepsilon}\left(u^{(s)}\right)(r) d w_{r}^{(s) k}-\int_{0}^{t} g^{\varepsilon}(u)(r) d w_{r}^{k}\right) \\
& I_{3}=E\left(\phi, \int_{0}^{t}\left(g^{\varepsilon}(u)(r)-g(u)(r)\right) d w_{r}^{k}\right)
\end{aligned}
$$


By the Cauchy-Schwarz inequality, (4.13), and (4.6) we have that $I_{1}$ converges to zero as $s \rightarrow \infty$. $I_{2}$ converges to zero by (4.16), and $I_{3}$ converges to zero by the CauchySchwarz inequality and (4.11). Hence, passing to the limit in (4.19) as $\varepsilon \rightarrow 0$ and $j \rightarrow \infty$, we get (4.18). This proves (4.8). (4.7) is then a consequence of (4.8) and (4.2).

Next we estimate the mathematical expectation of the expression in the left-hand side of (4.3). Setting $H_{s}=H_{1 s}+H_{2 s}$, where $H_{1 s}$ and $H_{2 s}$ are from the relation (3.7), with $u$ replaced by its approximation $u_{m}$, we have

$$
E \sum_{i=1}^{n} \int_{0}^{T} \int_{\Omega^{(s)}} \frac{\partial u^{(s)}}{\partial x_{i}} \frac{\partial h_{s}}{\partial x_{i}} d x d t=L_{1 s}+L_{2 s}+L_{3 s}
$$

where

$$
\begin{aligned}
L_{1 s} & =E \sum_{i=1}^{n} \int_{0}^{T} \int_{\Omega^{(s)}} \frac{\partial u^{(s)}}{\partial x_{i}} \frac{\partial h}{\partial x_{i}} d x d t \\
L_{2 s} & =E \sum_{i=1}^{n} \int_{0}^{T} \int_{\Omega^{(s)}} \frac{\partial H_{s}}{\partial x_{i}} \frac{\partial G_{s}}{\partial x_{i}} d x d t \\
L_{3 s} & =E \sum_{i=1}^{n} \int_{0}^{T} \int_{\Omega^{(s)}} \frac{\partial u_{m}}{\partial x_{i}} \frac{\partial G_{s}}{\partial x_{i}} d x d t+E \sum_{i=1}^{n} \int_{0}^{T} \int_{\Omega^{(s)}} \frac{\partial R_{s}}{\partial x_{i}} \frac{\partial G_{s}}{\partial x_{i}} d x d t .
\end{aligned}
$$

Since $u^{(s)}$ converges to $u$ weakly in $L_{2}\left(\Xi, L_{2}\left(0, T, H_{0}^{1}(\Omega)\right)\right)$ and $h \in H_{0}^{1}(\Omega)$, it follows that

$$
\lim _{s \rightarrow \infty} L_{1 s}=E \sum_{i=1}^{n} \int_{0}^{T} \int_{\Omega} \frac{\partial u}{\partial x_{i}} \frac{\partial h}{\partial x_{i}} d x d t
$$

By Theorem 3.2 (relation (3.13)) and (4.2) we get

$$
\lim _{s \rightarrow \infty} L_{3 s}=0 \text {. }
$$

We now estimate $L_{2 s}$. By the definition of $H_{s}, G_{s}, v_{i}^{(s)}$, and $\psi_{i}^{(s)}$, we have

$$
L_{2 s}=J_{1 s}+J_{2 s}+J_{3 s},
$$

where

$$
\begin{aligned}
J_{1 s} & =E \sum_{j=1}^{n} \sum_{i=1}^{I(s)} \int_{0}^{T} \int_{\Omega^{(s)}} \frac{\partial v_{i}^{(s)}}{\partial x_{j}} \frac{\partial}{\partial x_{j}}\left(v_{i}^{(s)} u_{m} h\left[\psi_{i}^{(s)}\right]^{2}\right) d x d t \\
J_{2 s} & =E \sum_{j=1}^{n} \sum_{i=1}^{I(s)} \int_{0}^{T} \int_{\Omega^{(s)}} v_{i}^{(s)} \frac{\partial}{\partial x_{j}}\left(u_{m} \psi_{i}^{(s)}\right) \frac{\partial}{\partial x_{j}}\left(v_{i}^{(s)} h \psi_{i}^{(s)}\right) d x d t \\
J_{3 s} & =E \sum_{j=1}^{n} \sum_{i=1}^{I(s)} \int_{0}^{T} \int_{\Omega^{(s)}} v_{i}^{(s)} h \psi_{i}^{(s)} \frac{\partial v_{i}^{(s)}}{\partial x_{j}} \frac{\partial}{\partial x_{j}}\left(u_{m} \psi_{i}^{(s)}\right) d x d t .
\end{aligned}
$$

We note that $J_{2 s}$ is a sum of expressions of the type

$$
E \sum_{j=1}^{n} \sum_{i=1}^{I(s)} \int_{0}^{T} \int_{\Omega^{(s)}} v_{i}^{(s)} \frac{\partial^{\alpha_{1}} u_{m}}{\partial x_{j}^{\alpha_{1}}} \frac{\partial^{\alpha_{2}} \psi_{i}^{(s)}}{\partial x_{j}^{\alpha_{2}}} \frac{\partial^{\alpha_{3}} v_{i}^{(s)}}{\partial x_{j}^{\alpha_{3}}} \frac{\partial^{\alpha_{4}} h}{\partial x_{j}^{\alpha_{4}}} \frac{\partial^{\alpha_{5}} \psi_{i}^{(s)}}{\partial x_{j}^{\alpha_{5}}} d x d t
$$


where the $\alpha_{k}$ 's are integers taking the values 0 or 1 and such that $\alpha_{1}+\alpha_{2}=1, \alpha_{3}+$ $\alpha_{4}+\alpha_{5}=1$. We shall prove that each of these expressions converges to zero. However, we limit ourselves to one of them; the others are proved analogously. We take for example

$$
J_{2 s}^{\prime}=E \sum_{j=1}^{n} \sum_{i=1}^{I(s)} \int_{0}^{T} \int_{\Omega^{(s)}}\left[v_{i}^{(s)}\right]^{2} u_{m}\left[\frac{\partial \psi_{i}^{(s)}}{\partial x_{j}}\right]^{2} h d x d t .
$$

Since $|h(x)| \leq C$ and $u_{m}$ is finite, we get

$$
J_{2 s}^{\prime} \leq C_{m} \sum_{i=1}^{I(s)}\left[\rho_{i}^{(s)}\right]^{-2} \int_{D_{i}^{(s)} \backslash B\left(x_{i}^{(s)}, \theta_{2} \rho_{i}^{(s)}\right)}\left[v_{i}^{(s)}(x)\right]^{2} d x
$$

where we have used the definition of $\psi_{i}^{(s)}(x)$. Thanks to (2.30), we have

$$
\begin{aligned}
J_{2 s}^{\prime} & \leq C_{m} \sum_{i \in I_{s}^{\prime}} \frac{\left[d_{i}^{(s)}\right]^{2(n-2)}}{\left[\rho_{i}^{(s)}\right]^{n-2}}+C_{m} \sum_{i \in I_{s}^{\prime \prime}} \frac{\left[d_{i}^{(s)}\right]^{2(n-2)}}{\left[\rho_{i}^{(s)}\right]^{n-2}} \\
& \leq C_{m} \sum_{i \in I_{s}^{\prime}}\left[d_{i}^{(s)}\right]^{n-2}+C_{m} \sup \left\{\frac{1}{\left[\ln r_{i}^{(s)}\right]^{2(n-2)}}\right\} \sum_{i=1}^{I(s)} \frac{\left[d_{i}^{(s)}\right]^{2(n-2)}}{\left[r_{i}^{(s)}\right]^{n}},
\end{aligned}
$$

where we have used the definition of the sets $I_{s}^{\prime}$ and $I_{s}^{\prime \prime}$. Passing to the limit in both sides of this inequality and taking into account the relation (3.2) and the condition (2.33) we get that $\lim _{s \rightarrow \infty} J_{2 s}^{\prime}=0$. Similar arguments show that the remaining expressions of the type (4.23) converge to zero. Therefore $\lim _{s \rightarrow \infty} J_{2 s}=0$. Analogously we prove that $\lim _{s \rightarrow \infty} J_{3 s}=0$.

Assuming that $s$ is sufficiently large so that $\theta_{1} \rho_{i}^{(s)}<1$, we can write

$$
J_{1 s}=J_{1 s}^{\prime}+J_{1 s}^{\prime \prime},
$$

where

$$
\begin{aligned}
& J_{1 s}^{\prime}=E \sum_{j=1}^{n} \sum_{i=1}^{I(s)} \int_{0}^{T} \int_{B_{i}^{(s)}} \frac{\partial v_{i}^{(s)}}{\partial x_{j}} \frac{\partial}{\partial x_{j}}\left[v_{i}^{(s)}(x) u_{m} h\left(\psi_{i}^{(s)}-1\right)\left(\psi_{i}^{(s)}+1\right)\right] d x d t, \\
& J_{1 s}^{\prime \prime}=E \sum_{j=1}^{n} \sum_{i=1}^{I(s)} \int_{0}^{T} \int_{\Omega^{(s)}} \frac{\partial v_{i}^{(s)}}{\partial x_{j}} \frac{\partial}{\partial x_{j}}\left[v_{i}^{(s)}(x) u_{m} h\right] d x d t .
\end{aligned}
$$

The function $\Phi_{i}^{(s)}(\omega, t, x)=v_{i}^{(s)}(x) u_{m} h\left(\psi_{i}^{(s)}-1\right)\left(\psi_{i}^{(s)}+1\right) \in H_{0}^{1}\left(B_{i}^{(s)}\right)$ for almost all $t$ and $\omega$. Thus by the definition of the functions $v_{i}^{(s)}(x)$ (it satisfies the integral identity (3.38)) we get that $J_{1 s}^{\prime}=0$.

Let us write

$$
J_{1 s}^{\prime \prime}=E \sum_{j=1}^{n} \sum_{i=1}^{I(s)} \int_{0}^{T} \int_{\Omega^{(s)}} \frac{\partial v_{i}^{(s)}}{\partial x_{j}} \frac{\partial v_{i}^{(s)}}{\partial x_{j}} u_{m} h d x d t
$$




$$
+E \sum_{j=1}^{n} \sum_{i=1}^{I(s)} \int_{0}^{T} \int_{\Omega^{(s)}} v_{i}^{(s)}(x) \frac{\partial v_{i}^{(s)}}{\partial x_{j}} \frac{\partial}{\partial x_{j}}\left[u_{m} h\right] d x d t .
$$

Arguing as we did above we can show that the second term in the right-hand side of (4.24) converges to zero. Thus

$$
J_{1 s}^{\prime \prime}=E \sum_{j=1}^{n} \sum_{i=1}^{I(s)} \int_{0}^{T} \int_{\Omega} \frac{\partial v_{i}^{(s)}}{\partial x_{j}} \frac{\partial v_{i}^{(s)}}{\partial x_{j}} u_{m} h d x d t+\mu_{s},
$$

where $\lim _{s \rightarrow \infty} \mu_{s}=0$.

We now cover $\Omega$ with a system of closed sets $G_{l}, l=1,2, \ldots, L$, such that the interior of $G_{l}$ does not meet the interior of $G_{k}$ for $l \neq k, \Omega=\cup_{l} G_{l}$, and furthermore $L$ is chosen such that for fixed $m$ the modulus of continuity of the function $u_{m} h$ in $G_{l}$ with respect to $x$ is less than $1 / m L$. We denote by $I_{s}\left(G_{l}\right)$ the set of indices $i=1,2, \ldots, I(s)$ such that $F_{i}^{(s)} \subset G_{l}$. Since the $F_{i}^{(s)}$ vanish as $s \rightarrow \infty$, we can choose $G_{l}$ so that $\cup_{l} I_{s}\left(G_{l}\right)=\{1,2, \ldots, I(s)\}$, i.e., all $F_{i}^{(s)}$ lie inside some $G_{l}$ for large $s$. Let $c(x)$ be the function defined in Hypothesis H3. We have

$$
\begin{aligned}
J_{1 s}^{\prime \prime}=E & \int_{0}^{T} \int_{\Omega} c(x) u(x) h(x) d x d t \\
& +E \sum_{l=1}^{L} \int_{0}^{T}\left\{\sum_{j=1}^{n} \sum_{i \in I_{s}\left(G_{l}\right)} \int_{G_{l}} \frac{\partial v_{i}^{(s)}}{\partial x_{j}} \frac{\partial v_{i}^{(s)}}{\partial x_{j}} d x-\int_{G_{l}} c(x) d x\right\} u_{m}\left(x_{i}^{(s)}\right) h\left(x_{i}^{(s)}\right) d t \\
& +E \sum_{l=1}^{L} \sum_{j=1}^{n} \int_{0}^{T} \sum_{i \in I_{s}\left(G_{l}\right)} \int_{G_{l}} \frac{\partial v_{i}^{(s)}}{\partial x_{j}} \frac{\partial v_{i}^{(s)}}{\partial x_{j}}\left(u_{m}(x) h(x)-u_{m}\left(x_{i}^{(s)}\right) h\left(x_{i}^{(s)}\right)\right) d x d t \\
& -E \sum_{l=1}^{L} \int_{0}^{T} \int_{G_{l}} c(x)\left(u_{m}(x) h(x)-u_{m}\left(x_{i}^{(s)}\right) h\left(x_{i}^{(s)}\right)\right) d x d t \\
& +E \sum_{l=1}^{L} \int_{0}^{T} \int_{G_{l}} c(x)\left(u_{m}(x)-u(x)\right) h d x d t+\mu_{s} ;
\end{aligned}
$$

we have omitted to write the variables $t$ and $\omega$ for simplicity. Passing to the limit in both sides of this equality first as $s \rightarrow \infty$ and later as $m \rightarrow \infty$, thanks to (2.34) and the properties of $u_{m}$ and $h$, we get by the dominated convergence theorem that

$$
\lim _{s \rightarrow \infty} J_{1 s}^{\prime \prime}=E \int_{0}^{T} \int_{\Omega} c(x) u h d x d t .
$$

As a result we have shown that

$$
\lim _{s \rightarrow \infty} L_{2 s}=E \int_{0}^{T} \int_{\Omega} c(x) u h d x d t .
$$

This together with the relations (4.4), (4.5), (4.7), (4.20), (4.21), and (4.22) enables us to pass to the limit in (4.3) and obtain that

$$
E \sum_{i=1}^{n} \int_{0}^{T} \int_{\Omega}\left[\frac{\partial u}{\partial x_{i}} \frac{\partial h}{\partial x_{i}}+c(x) u h\right] d x d t
$$




$$
\begin{aligned}
=-E & \int_{\Omega} u(\omega, T, x) h d x+E \int_{\Omega} u_{0}(x) h d x \\
& +E \int_{0}^{T} \int_{\Omega} f(t, u) h d x d t+E \sum_{k=1}^{d} \int_{0}^{T} \int_{\Omega} h g_{k}(t, u) d x d w_{t}^{k} .
\end{aligned}
$$

Since $h(x)$ is an arbitrary test function, this implies that $u(\omega, t, x)$ is a generalized solution of problem (2.35)-(2.37) for almost all $\omega$. This completes the proof of Theorem 2.8 .

Acknowledgments. The work was partly done at the Institute for Advanced Study in Princeton with the support of the National Science Foundation under the agreement No. DMS-0635607. The work also benefited from a research visit at the Isaac Newton Institute at Cambridge. The author expresses his deepest gratitude to these institutions for providing excellent conditions of work. The support of the National Research Foundation of South Africa is gratefully acknowledged.

\section{REFERENCES}

[1] J.E. Aarnes and Y. Efendiev, Mixed multiscale finite element methods for stochastic porous media flows, SIAM J. Sci. Comput., 30(5), 2319-2339, 2008.

[2] G. Allaire, Homogenization of the Navier-Stokes equations in open sets perforated with tiny holes. I. Abstract framework, Holes. Arch. Ratio. Mech. Anal., 113(3), 209-259, 1990.

[3] G. Allaire, Homogénéisation et convergence à deux échelles. Application à un problème de convection diffusion, C.R. Acad. Sci. Paris Sér. I Math., 312(8), 581-586, 1991.

[4] A. Bensoussan and R. Temam, Équations aux dérivées partielles stochastiques non linéaires. I, Israel J. Math., 11, 95-129, 1972.

[5] A. Bensoussan and R. Temam, Équations stochastiques du type Navier-Stokes, J. Funct. Anal., $13,195-222,1973$.

[6] A. Bensoussan, Homogenization of a class of stochastic partial differential equations, in Composite Media and Homogenization Theory, Birkhauser, Basel and Boston, MA, 47-65, 1991.

[7] A. Bensoussan, J.L. Lions, and G.C. Papanicolaou, Asymptotic Analysis for Periodic Structures, North-Holland, Amsterdam, 1978.

[8] A. Bensoussan, Some existence results for stochastic partial differential equations, in Stochastic Partial Differential Equations and Applications (Trento, 1990), Pitman Res. Notes Math. Ser., Longman Scientific and Technical, Harlow, UK, 268, 37-53, 1992.

[9] A. Bourgeat, A. Mikelić, and S. Wright, Stochastic two-scale convergence in the mean and applications, J. Reine Angew. Math., 456, 19-51, 1994.

[10] G. Bruno, A. Pankov, and T. Pankova, Time averaging for random nonlinear abstract parabolic equations, Abstr. Appl. Anal., 5(1), 1-11, 2000.

[11] L. Caffarelli and A. Mellet, Random homogenization of fractional obstacle problems, Netw. Heterog. Media, 3(3), 523-554, 2008.

[12] M.M. Cavalcanti, V.N. Domingos Cavalcanti, D. Andrade, and T.F. Ma, Homogenization for a nonlinear wave equation in domains with holes of small capacity, Disc. Cont. Dyn. Syst., 16(4), 721-743, 2006.

[13] D. Cioranescu, A. Damlamian, and G. Griso, The periodic unfolding method in homogenization, in Assyr, Banasiak, Damlamian, Sango: Multiple Scales Problems in Biomathematics, Mechanics, Physics and Numerics, GAKUTO Internat. Ser. Math. Sci. Appl., Gakkōtosho, Tokyo, 31, 1-35, 2009.

[14] D. Cioranescu and P. Donato, An Introduction to Homogenization, Oxford Lecture Series in Mathematics and its Applications, 17. The Clarendon Press, Oxford University Press, New York, 1999.

[15] D. Cioranescu, P. Donato, F. Murat, and E. Zuazua, Homogenization and corrector for the wave equation in domains with small holes, Ann. Scuola Norm. Sup. Pisa, 18, 251-293, 1991.

[16] D. Cioranescu and F. Murat, Un terme étrange venud'ailleurs. (French) [A strange term brought from somewhere else] Nonlinear partial differential equations and their applica- 
tions, Collège de France Seminar, Vol. II, 98-138, 389-390, Paris, 1979/1980; Res. Notes in Math., Pitman, Boston, Mass.-London, 60, 1982.

[17] G. Da Prato and J. Zabczyk, Stochastic Equations in Infinite Dimensions, in Encyclopedia of Mathematics and its Applications, Cambridge University Press, Cambridge, 44, 1992.

[18] G. Dal Maso and F. Murat, Asymptotic behaviour and correctors for Dirichlet problems in perforated domains with homogeneous monotone operators, Ann. Scuola Norm. Sup. Pisa Cl. Sci., (4)24, 2, 239-290, 1997.

[19] G. Dal Maso and L. Modica, Nonlinear stochastic homogenization and ergodic theory, J. Reine Angew. Math., 368, 28-42, 1986.

[20] Y. Efendiev and A. Pankov, Numerical homogenization of nonlinear random parabolic operators, Multiscale Model. Simul., 2(2), 237-268, 2004.

[21] Y. Efendiev, L. Jiang, and A. Pankov, Individual homogenization of nonlinear parabolic operators, Appl. Anal. 85(12), 1433-1457, 2006.

[22] L.C. Evans, Partial Differential Equations: Graduate Text in Mathematics, AMS, Providence, 1998.

[23] L.C. Evans and R.F. Gariepy, Measure Theory and Fine Properties of Functions, CRS Press, 1992.

[24] M. Freidlin, Functional Integration and Partial Differential Equations, Annals of Mathematics Studies, Princeton University Press, New Jersey, 109, 1985.

[25] I.I. Gikhman and A.V. Skorohod, Stochastic Differential Equations, Ergebnisse der Mathematik und ihrer Grenzgebiete, Band 72, Springer-Verlag, New York-Heidelberg, 1972.

[26] D. Gilbarg and N.S. Trudinger, Elliptic Partial Differential Equations of Second Order, Classics in Mathematics, Springer-Verlag, Berlin, 2001.

[27] N. Ichihara, Homogenization for stochastic partial differential equations derived from nonlinear filterings with feedback, J. Math. Soc. Japan, 57(2), 593-603, 2005.

[28] N. Ichihara, Homogenization problem for stochastic partial differential equations of Zakai type, Stoch. Rep., 76(3), 243-266, 2004.

[29] M. Kac, Probabilistic methods in some problems of scattering theory, Rocky Mountain J. Math., 4, 511-537, 1974.

[30] E.Y. Khruslov, The method of orthogonal projections and the Dirichlet problem in domains with fine-grained boundary, Math. USSR Sb., 17, 37-59 1972.

[31] E. Kosygina and S.R.S. Varadhan, Homogenization of Hamilton-Jacobi-Bellman equations with respect to time-space shifts in a stationary ergodic medium, Commun. Pure Appl. Math., 61(6), 816-847, 2008.

[32] O.A. Ladyzhenskaya and N.N. Uraltseva, Linear and Quasilinear Elliptic Equations, Academic Press, New-York, 1968.

[33] J.L. Lions and E. Magenes, Non-Homogenous Boundary Value Problems and Applications I, Springer-Verlag, Berlin - Heidelberg - New-York, 1972.

[34] J.L. Lions, Quelques méthodes de résolution des problèmes aux limites non linéaires, Dunod, Gauthiers-Villars, Paris, 1969.

[35] P-L. Lions and P.E. Souganidis, Homogenization of "viscous" Hamilton-Jacobi equations in stationary ergodic media, Commun. Part. Diff. Equ., 30(1-3), 335-375, 2005.

[36] P-L. Lions and P.E. Souganidis, Correctors for the homogenization of Hamilton-Jacobi equations in the stationary ergodic setting, Commun. Pure Appl. Math., 56(10), 1501-1524, 2003.

[37] S. Lototsky, Small perturbation of stochastic parabolic equations: A power series analysis, J. Funct. Anal., 193(1), 94-115, 2002.

[38] V.A. Marchenko and E.Y. Khruslov, Boundary Value Problems in Regions with Fine Grained Boundaries, Naukova Dumka, Kiev, 1974.

[39] V.A. Marchenko and E.Y. Khruslov, Homogenization of Partial Differential Equations, Progress in Mathematical Physics, Birkhäuser, Boston, 46, 2006.

[40] N. Nagase, Remarks on nonlinear stochastic partial differential equations: An application of the splitting-up method, SIAM J. Control \& Optim., 33, 1716-1730, 1995.

[41] G. Nguetseng, A general convergence result for a functional related to the theory of homogenization, SIAM J. Math. Anal., 20 (3), 608-623, 1989.

[42] B. Oksendal, Stochastic Differential Equations: An Introduction with Applications, Universitext, Springer-Verlag, Berlin-New York, 1998.

[43] A. Pankov, G-Convergence and Homogenization of Nonlinear Partial Differential Operators, Mathematics and its Applications, Kluwer Academic Publishers, Dordrecht, 422, 1997.

[44] G.C. Papanicolaou and S.R.S. Varadhan, Diffusions in regions with many small holes, Stochastic Differential Systems: Filtering and Control, Lecture Notes in Control and Information Sciences, Springer Verlag, Berlin, 25, 190-206, 1980. 
[45] G.C. Papanicolaou and S.R.S. Varadhan, Boundary value problems with rapidly oscillating random coefficients, Proceedings of a colloquium on random fields: Rigorous Results in Statistical Mechanics and Quantum Field Theory, Colloq. Math. Soc. J. Bolyai, NorthHolland, Amsterdam, 1979.

[46] E. Pardoux, Homogenization of linear and semilinear second-order parabolic partial differential equations with periodic coefficients: A probabilistic approach, J. Funct. Anal., 167, 498520, 1999

[47] Y.V. Prokhorov, Convergence of random processes and limit theorems in probability theory, Theory Prob. Appl., 1(2), 157-214, 1956.

[48] J. Rauch and M. Taylor, Potential and scattering theory, J. Funct. Anal., 18, 27-59, 1975.

[49] M. Sango, Magnetohydrodynamic turbulent flows: Existence results, Phys. D, 239(12), 912-923, 2010.

[50] M. Sango, Asymptotic behavior of a stochastic evolution problem in a varying domain, Stochastic Anal. Appl., 20(6), 1331-1358, 2002.

[51] M. Sango, Homogenization of the Dirichlet problem for a system of quasilinear elliptic equations in a domain with fine-grained boundary, Ann. Inst. H. Poincaré Anal. Non Linéaire, 20(2), 183-212, 2003.

[52] M. Sango, Asymptotic analysis of the initial boundary value problem for the thermoelastic system in a perforated domain, Colloq. Math., 95(1), 91-115, 2003.

[53] M. Sango, Pointwise a priori estimates for solution of a system of quasilinear elliptic equation, Appl. Anal., 80(3-4), 367-378, 2001.

[54] M. Sango and J-L. Woukeng, Stochastic two-scale convergence of an integral functional, Asymp. Anal., 73(1-2), 97-123, 2011.

[55] M. Sango and J-L. Woukeng, Stochastic $\Sigma$-convergence and applications, Dyn. Part. Diff. Equ., 8(4), 261-310, 2011.

[56] J. Simon, Compact sets in the space $L^{p}(0, T ; B)$, Annali Mat. Pura Appl., 146, IV, 65-96, 1987.

[57] A.V. Skorokhod, Limit theorems for stochastic processes, Teor. Veroyatnost. i Primenen., 1, 289-319, 1956. Society of London. A., 186, 123-164, 1895.

[58] I.V. Skrypnik, Methods for Analysis of Nonlinear Elliptic Boundary Value Problems, Nauka, Moscow, 1990. English translation in: Translations of Mathematical Monographs, AMS, Providence, 139, 1994.

[59] I.V. Skrypnik, Averaging of quasilinear parabolic problems in domains with fine-grained boundaries, (Russian) Differentsial'nye Uravneniya 31(2), 350-363, 368, 1995. Translation in Diff. Equ., 31(2), 327-339, 1995.

[60] Alain-Sol. Sznitman, Brownian Motion, Obstacles and Random Media, Springer Monographs in Mathematics, Springer-Verlag, Berlin, 1998.

[61] R. Temam, Navier-Stokes equations. Theory and Numerical Analysis, Studies in Mathematics and its Applications, Amsterdam-New York-Oxford, 2, 1977.

[62] W. Wang, D. Cao, and J. Duan, Effective macroscopic dynamics of stochastic partial differential equations in perforated domains, SIAM J. Math. Anal., 38(5), 1508-1527, 2006/07.

[63] W. Wang and J. Duan, Homogenized dynamics of stochastic partial differential equations with dynamical boundary conditions, Commun. Math. Phys., 275(1), 163-186, 2007.

[64] J. Wloka, Partial Differential Equations, Cambridge Univ. Press, 1987.

[65] V.V. Zhikov, S.M. Kozlov, and O.A. Oleinik, Homogenization of Differential Operators and Integral Functionals, Springer-Verlag, Berlin, 1994. 